\title{
Variable Consistency Dominance-Based Rough Set Approach to Preference Learning in Multicriteria Ranking
}

\author{
Marcin Szeląga,*, Salvatore Greco ${ }^{\mathrm{b}}$, Roman Słowiński ${ }^{\mathrm{a}, \mathrm{c}}$ \\ ${ }^{a}$ Institute of Computing Science, Poznań University of Technology, 60-965 Poznań, Poland \\ ${ }^{b}$ Department of Economics and Business, University of Catania, Corso Italia, 55, 95129 Catania, Italy and \\ Portsmouth Business School, Operations 85 Systems Management University of Portsmouth, Portsmouth PO1 3DE, UK \\ ${ }^{c}$ Systems Research Institute, Polish Academy of Sciences, 01-447 Warsaw, Poland
}

\begin{abstract}
We present a methodology for non-statistical preference learning in multicriteria ranking based on Variable Consistency Dominance-based Rough Set Approach (VC-DRSA). A finite set of objects to be ranked is evaluated by a set of criteria, which are real-valued functions with ordinal or cardinal scales. Given the statement of a multicriteria ranking problem, the only objective information one can get is the dominance relation over the set of objects. The dominance relation is, however, too poor because it leaves many objects incomparable. To enrich this relation, and make the objects more comparable, a decision maker (DM) must supply some preference information revealing her/his value system with respect to multicriteria evaluations. We are considering a frequent case, when the preference information has the form of pairwise comparisons of some objects relatively well known to the DM, called reference objects. This information is thus composed of some decision examples on the reference objects. It is the input data for a method that learns the preferences of the DM. Since this information is prone to inconsistencies, we propose to structure it using VC-DRSA. Then, the pairs of objects that are sufficiently consistent serve as a basis for induction of a preference model. This model has the form of a set of "if ..., then ..." decision rules. Application of these rules on the whole set of objects to be ranked yields a fuzzy preference structure (directed weighted graph). This preference structure is then exploited using a ranking method, so as to work out a final recommendation. We propose a list of properties that helps to choose a proper ranking method. The methodology is illustrated by an example.
\end{abstract}

Keywords: Multicriteria ranking, Decision rule, Dominance-based Rough Set Approach, Variable consistency, Ranking method, Pairwise comparison table

\section{Introduction}

\subsection{Ranking problem as a problem of Multicriteria Decision Aiding and Preference Learning}

In this paper, we present a methodology for dealing with a multicriteria ranking problem using a preference model in the form of a set of decision rules induced from decision examples. The ranking consists in ordering a set of objects (also called alternatives, solutions, acts, actions, options, candidates, ...) from the best to the worst, while these objects are evaluated from multiple points of view considered relevant for the problem at hand and called criteria (also called attributes, features, variables, ...). Multicriteria ranking problems constitute one of three main categories of decision problems considered in the field of Multiple Criteria Decision Aiding (also called Multiple Criteria Decision Making), which are ranking, choice and sorting (also called ordinal classification). As pointed out by Dembczyński et al. [28], Multicriteria Decision

\footnotetext{
* Corresponding author

Email addresses: mszelag@cs.put.poznan.pl (Marcin Szeląg), salgreco@unict.it (Salvatore Greco), rslowinski@cs.put.poznan.pl (Roman Słowiński)
} 
Aiding shares some goals, concepts and methodological issues with Preference Learning being an emerging subfield of Machine Learning. The main difference between them consists in the way of building a preference model of the Decision Maker (DM). While in Preference Learning (PL) the preference model results from statistical analysis of data (training examples), in Multicriteria Decision Aiding (MCDA) it is built from preference information elicited from the DM, very often interactively.

An important step in MCDA concerns selection and construction of criteria used for evaluation of objects. They are real-valued functions with ordinal or cardinal scales, built on elementary features of the objects. The aim is to set up a set of criteria which makes the pairwise comparison of all objects in the considered set meaningful. The criteria are equipped with monotonic preference scales which specify the preference orders in their value sets.

Remark that while in MCDA the construction of criteria with explicit monotonic preference scales is an important step in the procedure of decision aiding, in PL, the relationships between value sets of attributes and DM's preferences are discovered from data for a direct use in decision making. This means that in $\mathrm{PL}$, the monotonic preference scales converting elementary features to criteria are neither used nor revealed explicitly.

For a given finite set of objects $A$, and for a finite set of criteria $G=\left\{g_{1}, \ldots, g_{n}\right\}$ giving evaluations $g_{i}(a)$ to all $a \in A, i=1, \ldots, n$, the only objective information that comes out from comparison of these objects on multiple criteria is a dominance relation $D$ over set $A$. Given $a, b \in A$, object $a$ dominates object $b$, which is denoted by $a D b$, if and only if $a \succeq_{i} b$ for each $i=1, \ldots, n$, where $a \succeq_{i} b$ means that $g_{i}(a)$ "is at least as good as" $g_{i}(b)$. As $\succeq_{i}$ is a complete preorder over $A$, dominance relation $D$ is a partial preorder, i.e., a reflexive and transitive binary relation defined over $A$.

Apart from trivial cases, dominance relation $D$ is rather poor and leaves many objects incomparable (objects $a, b \in A$ are incomparable if neither $a D b$ nor $b D a$ ). In order to enrich the dominance relation and make the objects in $A$ more comparable, one needs additional information about value system of the DM, called preference information. This information permits to build a more or less explicit model of DM's preferences, called preference model. The preference model relates the decision to evaluations of the objects on the considered criteria. In other words, the preference model aggregates multicriteria evaluations of objects. It is inducing a preference structure on set $A$. A proper exploitation of this structure leads then to a recommendation in terms of ranking of objects from set $A$.

In PL, the training data are the equivalent of preference information in MCDA. Moreover, the aim of getting a preference model which permits to work out a final recommendation is the same for both methodologies - roughly speaking, the difference resides in statistical or non-statistical way of processing the preference information.

It follows from above that the preference information and the preference model are two crucial components of both MCDA and PL. The many methods existing in both fields differ just by these two components. Below, with respect to these two components, we review some recent trends in MCDA.

\subsection{Preference information and preference model}

As to the preference information, it depends on the adopted methodology: prices and interest rates for cost-benefit analysis, cost coefficients in objectives and technological coefficients in constraints for mathematical programming, a training set of decision examples for neural networks and machine learning, substitution rates for a value function of Multi-Attribute Utility Theory, pairwise comparisons of objects in terms of intensity of preference for the Analytic Hierarchy Process, attribute weights and several thresholds for ELECTRE methods, and so on (see the state-of-the-art survey by Figueira et al. [31]). This information has to be provided by the DM, possibly assisted by an analyst.

Very often the preference information is not easily definable. For example, this is the case of the price of many immaterial goods and of the interest rates in cost-benefit analysis, or the case of the coefficients of objectives and constraints in mathematical programming models. Even if the required information is easily definable, like a training set of decision examples for neural networks, it is often processed in a way which is not clear for the DM, such that (s)he cannot see what are the exact relations between the provided information and the final recommendation. Consequently, very often the decision aiding method 
is perceived by the DM as a black box whose result has to be accepted because the analyst's authority guarantees that the result is "right". In this context, the aspiration of the DM to find good reasons to make decision is frustrated and raises the need for a more transparent methodology in which the relation between the original information and the final recommendation is clearly shown. Such a transparent methodology searched for has been called glass box [51]. Its typical representative is using a training set of decision examples as the input preference information.

The decision examples may either by provided by the DM on a set of real or hypothetical objects, or may come from observation of DM's past decisions. Such an approach follows the paradigm of inductive learning used in artificial intelligence [65], or robust ordinal regression becoming popular in operational research [56]. It is also concordant with the principle of posterior rationality postulated by March [64] since it emphasizes the discovery of DM's intentions as an interpretation of actions rather than as a priori position. This paradigm has been used to construct various preference models from decision examples, e.g., the general additive utility functions [32, 54], the outranking relations [41, 66], the monotonic decision trees [40], and the set of "if ..., then ..." decision rules [48].

Of particular interest is the last model based on decision rules - it has been introduced to decision analysis by Greco, Matarazzo and Słowiński [44, 46, 85]. A popular saying attributed to Slovic [81] is that "people make decisions and then search for rules that justify their choices". The rules explain the preferential attitude of the DM and enable understanding of the reasons of his/her past decisions. The recognition of the rules by the DM [63] justifies their use for decision support. So, the preference model in the form of rules derived from decision examples fulfills both explanation and recommendation goals of decision aiding.

For example, in case of ranking students at the end of their studies, the decision rule approach requires as input information a set of examples of pairwise comparisons of some students, taking into account their evaluations on the considered criteria (courses, projects). From these pairwise comparisons, some decision rules are induced, such as "if student $a$ is strongly preferred to student $b$ on Mathematics, and student $a$ got at least 75/100 for the Decision Support Project while student $b$ got at most 80/100 for the same project, then $a$ is weakly preferred to $b$ ". Each one of such rules is directly related to examples of pairwise comparisons in the input information.

The traditional preference models, which are the utility function and the outranking relation, can be represented in terms of equivalent decision rules. The clarity of the rule representation of preferences enables one to see the limits of these aggregation functions. Several studies [47, 49, 83] presented an axiomatic characterization of all three kinds of preference models in terms of conjoint measurement theory and in terms of a set of decision rules. The given axioms of "cancellation property" type are the weakest possible. In comparison to other studies on the characterization of preference models, these axioms do not require any preliminary assumptions about the scales of preferences of criteria. A side-result of these investigations is that the decision rule preference model is the most general among all known models.

\subsection{Dominance-based Rough Set as a tool for dealing with inconsistency of the preference information}

Preference information given in terms of decision examples is often inconsistent. This explains the interest in rough set theory proposed by Pawlak [67-69]. Rough set theory permits to structure the data set such that sets of objects (or sets of pairs of objects) are represented by pairs of ordinary sets called lower and upper approximations. The differences between upper and lower approximations are called boundary sets, and their cardinalities indicate to what degree the data set is inconsistent. Induction of decision rules from data structured in this way permits to obtain certain or possible decision rules [70, 82].

As the classical definition of rough sets is based on an indiscernibility relation in the set of objects [67-69], it cannot handle inconsistency encountered in decision examples involving multicriteria evaluations. To deal with this kind of inconsistency, Greco, Matarazzo and Słowiński generalized the classical rough set approach, so as to take into account preference orders and monotonic relationships between evaluations on criteria and assignment to decision classes. This generalization, called Dominance-based Rough Set Approach (DRSA), has been adapted to a large variety of decision problems, including the multicriteria ranking problem [35, 44, 46, 51, 52, 84, 85].

The usefulness of DRSA goes beyond the frame of MCDA. This is because the type of monotonic relationships handled by DRSA is also meaningful for problems where preferences are not considered but 
a kind of monotonicity relating ordered attribute values is meaningful for the analysis of data at hand. Indeed, monotonicity concerns, in general, mutual trends existing between different variables, like distance and gravity in physics, or inflation rate and interest rate in economics [50]. Whenever a relationship between different aspects of a phenomenon is discovered, this relationship can be represented by a monotonicity with respect to some specific measures or perception of the considered aspects, e.g., "the colder the weather, the higher the energy consumption" or "the more a tomato is red, the more it is ripe". The qualifiers, like "cold weather", "high energy consumption", "red" and "ripe", may be expressed either in terms of some measurement units, or in terms of degrees of membership to fuzzy sets representing these concepts. Note, moreover, that DRSA can be adapted to discover rules from any kind of input data, exhibiting monotonic relationships which are unknown a priori and hold in some parts of the evaluation space only. This requires a proper non-invasive transformation of the data, permitting representation of both positive and negative monotonic relationships that are to be discovered [8].

Recently, we have been able to observe an increasing interest in statistical methods of processing preference information concerning multicriteria decision problems, particularly in situations where the number of decision examples is very large. Statistical approach to learning preference models from decision examples is the core of PL. This interest is motivated by new challenging applications related to Internet, in particular, recommender systems and information retrieval. In the first, the task is to recommend to the user a new item (like movie or book) that fits her/his preferences. The recommendation is computed on the basis of the learning information describing the past behavior of the user. In the latter, the task is to sort (or rank) the documents retrieved by the search engine according to the user's preferences. There are several algorithms that are tailored for these kinds of problems. The most popular are based on rank loss minimization. These include variants of support vector machines [61] and boosting [38]. One should also note that there exist several other learning approaches in which preferences are modeled [37, 39, 74, 86]. Moreover, an interesting work has been done in the field of ordinal classification with monotonicity constraints [25-27, 62].

\subsection{Content and plan of the paper}

In this paper, we present a non-statistical methodology for preference learning from decision examples. It employs an adaptation of DRSA to the multicriteria ranking problem [42-44, 46, 84, 85]. More precisely, it employs an adaptation of a generalized version of DRSA called Variable Consistency Dominance-based Rough Set Approach (VC-DRSA) [11]. VC-DRSA is a probabilistic version of DRSA, however, it is not a statistical preference learning methodology in the sense of statistical machine learning, where the preference model is learned so as to minimize a loss function admitted for parameter estimation over a training set. In the current adaptation, decision examples have the form of pairwise comparisons of some reference objects. These pairwise comparisons, presented in a so-called pairwise comparison table (PCT), specify if a weak preference relation, called an outranking relation $S$, holds for the considered pairs of reference objects or not. When weak preference relation does not hold, such a relation is called a non-outranking relation $S^{c}$. Thus, decision rules induced from rough approximations of comprehensive preference relations $S$ and $S^{c}$ also involve pairs of objects. They constitute the preference model of the DM who gave the pairwise comparisons. Application of these rules on a set $A$ of objects to be ranked yields a fuzzy preference structure on $A$, represented by a directed weighted graph. In order to pass from the preference structure to the recommended ranking of objects, one has to apply an exploitation procedure including a ranking method. In this paper, we investigate properties of several ranking methods which are supposed to be useful. From among these methods, we choose the one that enjoys the most desirable properties.

This paper extends our considerations from [87] where the preference structure induced by decision rules was supposed to be crisp. Passing from crisp to fuzzy preference structure on the set of objects requires a proper adaptation of the exploitation procedure. Moreover, the properties of ranking methods change in case of fuzzy preference structures. Another important difference between this paper and [87] concerns the set of evaluation criteria. In [87], we adopted a typical assumption of MCDA that the set of criteria is a consistent family [78], i.e., we assumed that $G$ satisfies the properties of completeness (all relevant criteria are considered), monotonicity (the better the evaluation of an object on considered criteria, the more it is preferable to another object), and non-redundancy (there is no criterion which could be removed without 
violating one of the previous two properties). In this paper, we drop this assumption, which is rather typical for PL. This drop is reflected by a different way of constructing the preference structure.

The remainder of this paper is organized as follows. Section 2 concerns some basic notions and notation used throughout the paper. In Section 3, we present our setting of the considered multicriteria ranking problem. In Section 4, we introduce the concept of the PCT. Section 5 concerns rough approximation of two comprehensive preference relations specified by a DM - outranking relation $S$ and non-outranking relation $S^{c}$. In Section 6, we discuss induction of decision rules from rough approximations of $S$ and $S^{c}$. Section 7 concerns application of decision rules on a set of objects to be ranked. In Section 8, we describe exploitation of the preference structure resulting from application of decision rules. In this section, we focus on some desirable properties of several ranking methods and select the method that has the best properties. In Section 9, we present an illustrative example that demonstrates usefulness of the proposed methodology. Section 10 concludes the paper.

\section{Preliminaries}

In this paper, $A$ will denote a finite set of objects to be ranked. A fuzzy (valued) relation $R$ over $A$ is a function from $A \times A$ into [0,1]. It is said to be reflexive if $R(a, a)=1$, for all $a \in A$. It is said to be irreflexive if $R(a, a)=0$, for all $a \in A$. We denote by $\mathbf{R}_{A}$ the set of all fuzzy relations over $A$. Moreover, we denote by $R / A^{\prime}$ the restriction of fuzzy relation $R$ over $A$ to set $A^{\prime} \subseteq A$, i.e., fuzzy relation over $A^{\prime}$ such that for all $a, b \in A^{\prime}, R / A^{\prime}(a, b)=R(a, b)$. A fuzzy relation $R$ over $A$ such that $R(a, b) \in\{0,1\}$, for all $a, b \in A$, is said to be crisp. In such case:

- if $R(a, b)=1$, we say that pair $(a, b)$ belongs to relation $R$, and we write $a R b$ or $(a, b) \in R$,

- if $R(a, b)=0$, we say that pair $(a, b)$ does not belong to relation $R$, and we write not $a R b$ or $(a, b) \notin R$.

Let $R$ be a crisp relation over $A$. This relation is said to be:

- transitive if $(a R b$ and $b R c \Rightarrow a R c)$,

- complete if $(a R b$ or $b R a)$,

for all $a, b, c \in A$.

A weak order (also called complete preorder or total preorder) over $A$ is a crisp binary relation which is reflexive, transitive, and complete. A partial preorder over $A$ is a crisp binary relation which is reflexive, and transitive, but non-complete, in general. The symmetric part of a weak order relation $R$ yields equivalence classes ordered by the asymmetric part of $R$.

Let $R$ be a crisp relation over $A$. We denote by $G(A, R)$ the set of greatest elements of $A$ given $R$, i.e.,

$$
G(A, R)=\{a \in A: a R b \text { for all } b \in A \backslash\{a\}\} .
$$

It should be noticed that $G(A, R)$ may well be empty. When $R$ is a weak order, it is easy to see that set $G(A, R)$ is non-empty and equal to the first equivalence class of $R$.

A ranking method $(\mathrm{RM}) \succeq$ is a function assigning a partial preorder $\succeq(A, R)$ over $A$ to any finite set $A$ and any fuzzy relation $R$ over this set. Remark that this is an extended definition w.r.t. the one given by Bouyssou and Vincke [22], Bouyssou and Pirlot [21], and Greco et al. [53], where $\succeq(A, R)$ was supposed to be a weak order. Moreover, in $[21,22] \succeq$ was called a ranking rule. However, in this paper, we call $\succeq$ a ranking method to avoid confusion with decision rules.

We, respectively, denote by $=(A, R)$ and $\succ(A, R)$ the symmetric and asymmetric parts of $\succeq(A, R)$, i.e., the relations such that, for all $a, b \in A$,

$$
\begin{aligned}
& a=(A, R) b \Leftrightarrow a \succeq(A, R) b \text { and } b \succeq(A, R) a, \\
& a \succeq(A, R) b \Leftrightarrow a \succeq(A, R) b \text { and not } b \succeq(A, R) a .
\end{aligned}
$$




\section{Problem Setting}

We consider a multicriteria ranking problem in which objects belonging to a finite set $A$ have to be ranked, either completely or partially. In the first case, one aims at obtaining a weak order over $A$. In the latter case, one accepts a partial preorder over $A$. The objects from set $A$ are evaluated by set $G=\left\{g_{1}, \ldots, g_{n}\right\}$ of $n$ criteria. Each criterion $g_{i} \in G, i=1, \ldots, n$, is a real-valued function $g_{i}: A \rightarrow \Re$, with cardinal (i.e., interval or ratio) scale or ordinal scale (which is given a priori or results from an order-preserving number-coding of non-numerical ordinal evaluations) $[45,80]$. Thus, value $g_{i}(a), a \in A$, represents the evaluation of object $a$ with respect to (w.r.t.) criterion $g_{i}$. A criterion with the cardinal scale is called a cardinal criterion; the set of all cardinal criteria is denoted by $G^{N} \subseteq G$. A criterion with the ordinal scale is called an ordinal criterion; the set of all ordinal criteria is denoted by $G^{O} \subseteq G$. Moreover, $G^{N} \cup G^{O}=G$ and $G^{N} \cap G^{O}=\emptyset$. The meaning of the two scales is such that in the case of a criterion $g_{i} \in G^{N}$ with a cardinal scale, one can define a function $k_{i}: \Re^{2} \rightarrow \Re$ which measures the intensity of preference (positive or negative) of object $a$ over object $b$, taking into account evaluations $g_{i}(a), g_{i}(b), a, b \in A$. For properties of function $k_{i}$, and different ways of defining it, see Greco et al. [46]. Basically, $k_{i}$ is non-decreasing w.r.t. the first argument, and non-increasing w.r.t. the second argument. For the sake of simplicity, we assume in this paper that for each cardinal criterion $g_{i} \in G^{N}$, intensity of preference is defined as: $k_{i}\left[g_{i}(a), g_{i}(b)\right]=\Delta_{i}(a, b)=g_{i}(a)-g_{i}(b)$. In the case of a criterion $g_{i} \in G^{O}$ with an ordinal scale, this is not possible (as differences of evaluations are not meaningful) and one can only establish an order of evaluations $g_{i}(a), a \in A$.

We assume, moreover, without loss of generality, that all the criteria are of gain-type, i.e., the greater the criterion value the better.

Let us denote by $V_{g_{i}}=\Re$ the value set (domain) of criterion $g_{i} \in G$. Then, set $V_{G}=\prod_{i=1, \ldots, n} V_{g_{i}}=\Re^{n}$ is called $G$-evaluation space.

Given the statement of a multicriteria ranking problem, the only objective information one can get is the dominance relation $D$ over set of objects $A$, defined in the $G$-evaluation space. Let us consider objects $a, b \in A$; object $a$ is said to dominate object $b$, denoted by $a D b$, if and only if (iff) for all $g_{i} \in G: g_{i}(a) \geq g_{i}(b)$. The dominance relation $D$ is, however, too poor because it leaves many objects incomparable. In order to make the objects more comparable, a DM must supply some preference information revealing her/his value system w.r.t. multicriteria evaluations. We consider a frequent case, when the preference information has the form of pairwise comparisons of some objects relatively well known to the DM, called reference objects. This information is thus composed of some decision examples on the reference objects.

Let us denote by $A^{R}$ the set of all reference objects. Set $A^{R}$ can be a subset of $A$, however, it is not required by the presented methodology. If $A^{R} \nsubseteq A$, then we just need to define each criterion $g_{i} \in G, i=$ $1, \ldots, n$, as function $g_{i}: A \cup A^{R} \rightarrow \Re$, and dominance relation $D$ over set $A \cup A^{R}$. In any case, $A \backslash A^{R}$ is a set of objects unseen during preference model learning.

Following Greco et al. [44, 46] and Słowiński et al. [85], we consider that for each ordered pair $(a, b)$ of different reference objects, i.e., $(a, b) \in A^{R} \times A^{R}, a \neq b$, the DM can state either "object $a$ is at least as good as object $b$ " (in other words - "object $a$ outranks object $b$ ") or "object $a$ is not at least as good as object $b$ " (in other words - "object $a$ does not outrank object $b$ "), or abstain from any judgment. The first situation is denoted by $a S b$ (or $(a, b) \in S$ ), while the second one is denoted by $a S^{c} b$ (or $(a, b) \in S^{c}$ ). Moreover, we fix $a S a$ for all $a \in A^{R}$. Thus, from a formal point of view, the DM can reveal her/his preferences by assigning pairs of objects to any of the two considered comprehensive preference relations: outranking relation $S$ or non-outranking relation $S^{c}$. Obviously, relation $S$ is a weak preference relation which, in general, is only reflexive. It is not symmetric, not transitive, and not complete. Moreover, non-outranking relation $S^{c}$ is irreflexive, and, in general, it is not symmetric, not transitive, and not complete. This is to say that the preference information coming from the DM is relatively weak and non-exhaustive.

By expressing her/his preferences in the way described above, for each pair of objects $(a, b) \in A^{R} \times A^{R}$, $a \neq b$, the DM can easily specify any of the four situations typically considered in Multiple Criteria Decision Aiding (MCDA), i.e.:

- strict preference P:

$$
a P b \Leftrightarrow a S b \wedge b S^{c} a
$$


- inverse strict preference $P^{-1}$ :

$$
a P^{-1} b \Leftrightarrow a S^{c} b \wedge b S a,
$$

- indifference I:

$$
a I b \Leftrightarrow a S b \wedge b S a,
$$

- incomparability $J$ :

$$
a J b \Leftrightarrow a S^{c} b \wedge b S^{c} a .
$$

Alternative elicitation of preference information in terms of pairwise comparisons employing graded comprehensive preference relations can be found in [35].

It is worth stressing that expressing decision examples on the reference objects is cognitively relatively easy for the DM. In our approach, instead of requiring that the DM provides values of some difficult parameters like weights of criteria or different thresholds (see, e.g., methods from the ELECTRE family [76]), and then using this information in a complex preference model, we treat the decision examples supplied by the DM as the input data, and then follow with learning of a preference model of the DM in easy rule terms.

To simplify the notation, in the following, we will use unique symbol $T$ to refer to any of the comprehensive preference relations $S$ and $S^{c}$ when these relations are considered jointly, unless this may cause misunderstanding. Moreover, we denote by $\mathcal{I}_{G}, \mathcal{I}_{G^{N}}, \mathcal{I}_{G^{O}} \subseteq\{1, \ldots, n\}$ the sets of indexes of criteria belonging to $G, G^{N}, G^{O}$, respectively, where $\mathcal{I}_{G^{N}} \cap \mathcal{I}_{G^{O}}=\emptyset$ and $\mathcal{I}_{G^{N}} \cup \mathcal{I}_{G^{O}}=\mathcal{I}_{G}$.

\section{Pairwise Comparison Table}

The preference information of the DM in the form of pairwise comparisons of reference objects is used to create a pairwise comparison table (PCT), first introduced in [42, 43]. Let us denote by $B \subseteq A^{R} \times A^{R}$ the set of pairs of reference objects for which the comprehensive preference of the DM is known. This set is composed of pairs $(a, b) \in A^{R} \times A^{R}, a \neq b$, for which the DM expressed her/his preferences by declaring $a S b$ or $a S^{c} b$, as well as of pairs $(a, a), a \in A^{R}$, which are assigned to $S$.

Intuitively, a PCT created on the basis of preference information supplied by the DM is an $m \times(n+1)$ data table, denoted by $S_{P C T}$, where $m$ is the cardinality of set $B$ of pairs. First $n$ columns of this table correspond to criteria from set $G$. The last, $(n+1)$-th, column represents the comprehensive preference relation $S$ or $S^{c}$. Each row of $S_{P C T}$ corresponds to a pair of reference objects from $B$. As announced in Section 3, when comparing two objects $a, b \in A^{R}$ on a cardinal criterion $g_{i} \in G^{N}, i \in \mathcal{I}_{G^{N}}$, one puts in the corresponding column of $S_{P C T}$ the difference $g_{i}(a)-g_{i}(b)$. When comparing two objects $a, b \in A^{R}$ on an ordinal criterion $g_{i} \in G^{O}, i \in \mathcal{I}_{G^{O}}$, one puts in the corresponding column of $S_{P C T}$ an ordered pair of ordinal evaluations $\left(g_{i}(a), g_{i}(b)\right)$.

Describing the PCT more formally, one can say that each pair of objects $(a, b) \in B$ is evaluated on set $G$ of criteria, such that:

- for criterion $g_{i} \in G^{N}$, the evaluation of $(a, b) \in B$ is defined as $q_{i}(a, b)=g_{i}(a)-g_{i}(b) \in V_{q_{i}}=\Re$,

- for criterion $g_{i} \in G^{O}$, the evaluation of $(a, b) \in B$ is defined as $q_{i}(a, b)=\left(g_{i}(a), g_{i}(b)\right) \in V_{q_{i}}=\Re \times \Re$.

Then, set $V_{Q}=\prod_{i \in \mathcal{I}_{G}} V_{q_{i}}$ is called $Q$-evaluation space. 


\section{Rough Approximation of Outranking and Non-outranking Relations}

In Section 3, we considered dominance relation $D$ over set of objects $A$, defined in the $G$-evaluation space. Here, we introduce another type of dominance relation, denoted by $D_{2}$. This binary relation is defined over set $B$ of pairs of objects, in the $Q$-evaluation space. $D_{2}$ can also be defined using the evaluations of objects from set $A^{R}$ on the criteria from set $G$. Obviously, the same definition holds for all non-empty subsets of $G$.

First, let us consider a case when set $G$ is composed of cardinal criteria only, i.e. $G^{N}=G, G^{O}=\emptyset$. Then, given pairs of objects $(a, b),(c, d) \in B$, pair $(a, b)$ is said to dominate pair $(c, d)$ w.r.t. criteria from $G$ (denoted by $\left.(a, b) D_{2}(c, d)\right)$ iff $\Delta_{i}(a, b) \geq \Delta_{i}(c, d)$ for each $g_{i} \in G$, where $\Delta_{i}(a, b)$ denotes $g_{i}(a)-g_{i}(b)$. Let $D_{2}^{i}$ be the dominance relation over $B$ confined to single criterion $g_{i} \in G$. This relation is reflexive, transitive and complete. Therefore, $D_{2}^{i}$ is a weak order over $B$. Since an intersection of weak orders is a partial preorder, and relation $D_{2}$ over $B$ is the intersection of relations $D_{2}^{i}, i \in \mathcal{I}_{G}$, then relation $D_{2}$ is a partial preorder over $B$.

Secondly, let us consider a case when set $G$ is composed of ordinal criteria only, i.e., $G^{O}=G, G^{N}=\emptyset$. Then, given pairs of objects $(a, b),(c, d) \in B$, pair $(a, b)$ is said to dominate pair $(c, d)$ w.r.t. criteria from $G$ iff $g_{i}(a) \geq g_{i}(c)$ and $g_{i}(b) \leq g_{i}(d)$ for each $g_{i} \in G$. In other words, pair $(a, b)$ is said to dominate pair $(c, d)$ w.r.t. criteria from $G$ iff $a D c$ and $d D b$. Let $D_{2}^{i}$ be the dominance relation over $B$ confined to single criterion $g_{i} \in G$. This relation is reflexive, transitive but non-complete (i.e., it is possible that not $(a, b) D_{2}^{i}(c, d)$ and not $(c, d) D_{2}^{i}(a, b)$ for some $(a, b),(c, d) \in B$ and $\left.g_{i} \in G\right)$. Therefore, $D_{2}^{i}$ is a partial preorder over $B$. Since an intersection of partial preorders is also a partial preorder, and relation $D_{2}$ over $B$ is the intersection of relations $D_{2}^{i}, i \in \mathcal{I}_{G}$, then relation $D_{2}$ is a partial preorder over $B$.

Finally, when set $G$ is composed of both cardinal and ordinal criteria, i.e., when $G^{N} \neq \emptyset$ and $G^{O} \neq \emptyset$, then given pairs of objects $(a, b),(c, d) \in B$, pair $(a, b)$ is said to dominate pair $(c, d)$ w.r.t. criteria from $G$ iff $(a, b)$ dominates $(c, d)$ w.r.t. both $G^{N}$ and $G^{O}$. Since the dominance w.r.t. $G^{N}$ is a partial preorder over $B$ and the dominance w.r.t. $G^{O}$ is a partial preorder over $B$, then the dominance $D_{2}$, being the intersection of these two dominance relations, is also a partial preorder over $B$.

Let $G^{\prime} \subseteq G$ and pairs $(a, b),(c, d) \in B$. Then, if $(a, b)$ dominates $(c, d)$ w.r.t. set $G$ of criteria, then $(a, b)$ dominates $(c, d)$ w.r.t. set $G^{\prime}$.

Given a pair of objects $(a, b) \in B$ we define the following:

- a set of pairs of objects dominating $(a, b)$, called the dominating set or positive dominance cone in the $Q$-evaluation space:

$$
D_{2}^{+}(a, b)=\left\{(c, d) \in B:(c, d) D_{2}(a, b)\right\},
$$

- a set of pairs of objects dominated by $(a, b)$, called the dominated set or negative dominance cone in the $Q$-evaluation space:

$$
D_{2}^{-}(a, b)=\left\{(c, d) \in B:(a, b) D_{2}(c, d)\right\} .
$$

In equations (8) and (9), pair of objects $(a, b)$ is called an origin of the dominance cone. Dominating and dominated sets of objects are "granules of knowledge" used to approximate outranking and non-outranking relation, respectively.

We formulate the following dominance principle w.r.t. pairwise comparisons of the DM: "if $a$ is preferred to $b$ at least as much as $c$ is preferred to $d$ w.r.t. each $g_{i} \in G$, then the comprehensive preference of $a$ over $b$ should not be weaker that the comprehensive preference of $c$ over $d "$. This means that if $(a, b) D_{2}(c, d)$, one expects that:

(i) if $a S^{c} b$, then $c S^{c} d$,

(ii) if $c S d$, then $a S b$.

Violation of this dominance principle is considered as an inconsistency w.r.t. dominance relation $D_{2}$ over $B$. Let us observe that, thanks to the presence in $S_{P C T}$ of pairs $(a, a) \in S, a \in A^{R}$, an inconsistency w.r.t. $D_{2}$ appears also when given two objects $a, b \in A^{R}$, the DM states that $a S^{c} b$, while $a D b$. This is related to the 
reflexivity of $S$ and the irreflexivity of $S^{c}$. In fact, $a D b$ implies $(a, b) D_{2}(a, a)$, and together with $a S a$, this implies that $a S b$. Thus, the opposite statement $a S^{c} b$ is inconsistent with the expectation (ii) listed above.

In practice, decision examples given by a DM are often inconsistent due to hesitation of the DM, unstable character of her/his preferences, or incomplete determination of the set of criteria [e.g., 77]. These inconsistencies cannot be considered as a simple error or as noise. They can convey important information that should be taken into account when constructing a preference model of the DM. Rather than correct or ignore these inconsistencies, we propose to handle them using the dominance-based rough set approach. Before learning of a preference model of the DM, we structure pairs of objects contained in $S_{P C T}$ by calculation of lower approximations of comprehensive preference relations. In this way, we restrict a priori the set of pairs of objects on which the preference model is built to a subset of sufficiently consistent pairs of objects belonging to lower approximations. This restriction is motivated by a postulate for learning from (sufficiently) consistent data, so that the knowledge gained from this learning is relatively certain (or, in other words, the induced preference model is reliable). Analogous restriction proved to be effective in case of ordinal classification problems with monotonicity constraints $[12,13]$. It is worth underlining that, although only sufficiently consistent pairs of objects from $S_{P C T}$ are used to construct a preference model of the DM, the remaining pairs of objects are not removed from $S_{P C T}$. In other words, the approach proposed in this paper does not boil down to a simple pre-processing performed to remove inconsistent decision examples. In fact, inconsistent pairs of objects play the role of "counterexamples", helping this way to induce a preference model.

In previous applications of DRSA to multicriteria ranking [44, 46, 85], outranking and non-outranking relations were approximated using strict inclusion relation between dominance cones originating in pairs of objects $(a, b) \in B$ and the comprehensive preference relations. Precisely, lower approximations of relations $S$ and $S^{c}$ were defined as:

$$
\begin{aligned}
\underline{S} & =\left\{(a, b) \in B: D_{2}^{+}(a, b) \subseteq S\right\}, \\
\underline{S^{c}} & =\left\{(a, b) \in B: D_{2}^{-}(a, b) \subseteq S^{c}\right\} .
\end{aligned}
$$

These definitions of lower approximations appear to be too restrictive in practical applications. In consequence, lower approximations of $S$ and $S^{c}$ are often empty, preventing generalization of pairwise comparisons in terms of sufficiently certain decision rules. Therefore, in this paper, we apply VC-DRSA [11, 58] which is a probabilistic extension of the classical DRSA. Since originally VC-DRSA was introduced for ordinal classification problems, here we adapt its definitions of variable-consistency (v-c) lower approximations to the case of approximating outranking and non-outranking relations. In the adapted definitions of v-c lower approximations of $S$ and $S^{c}$, we use consistency measure $\epsilon_{T}: B \rightarrow[0,1]$, introduced in [10, 11], defined as:

$$
\begin{aligned}
& \epsilon_{S}(a, b)=\frac{\left|D_{2}^{+}(a, b) \cap S^{c}\right|}{\left|S^{c}\right|}, \\
& \epsilon_{S^{c}}(a, b)=\frac{\left|D_{2}^{-}(a, b) \cap S\right|}{|S|} .
\end{aligned}
$$

Given pair of objects $(a, b) \in B$ and relation $T$, value $\epsilon_{T}(a, b)$ reflects consistency of pair $(a, b)$ w.r.t. $T$. $\epsilon_{T}$ is a cost-type consistency measure, which means that value zero denotes full consistency and the greater the value, the less consistent is a given pair of objects. The definitions of v-c lower approximations adapted to the case of approximating outranking and non-outranking relations are the following:

$$
\begin{aligned}
\underline{S} & =\left\{(a, b) \in S: \epsilon_{S}(a, b) \leq \theta_{S}\right\}, \\
\underline{S^{c}} & =\left\{(a, b) \in S^{c}: \epsilon_{S^{c}}(a, b) \leq \theta_{S^{c}}\right\},
\end{aligned}
$$

where consistency thresholds $\theta_{S}, \theta_{S^{c}} \in[0,1)$. The values of these thresholds are fixed using a simple wrapper-like cross validation procedure. Note that in case $\theta_{S}=\theta_{S^{c}}=0$, the v-c lower approximations (14) and (15) are equal to the lower approximations (10) and (11), respectively. In the following, unless this may cause misunderstanding, we drop "v-c" and call sets of pairs of objects defined by (14) and (15) just lower approximations of relations $S$ and $S^{c}$, respectively. 
In [11], several consistency measures were defined. The choice of particular consistency measure $\epsilon_{T}$ is dictated by several factors. The first one is that measure $\epsilon_{T}$ features an easy interpretation - it can be interpreted as an estimate of conditional probability that a pair of objects $(c, d) \in B$ belongs to the dominance cone originating in pair $(a, b) \in B$ given that pair $(c, d)$ does not belong to the considered comprehensive preference relation. The second factor is a good performance of this measure in computational experiments $[12,13]$, comparing to other consistency measures. The third factor is the fact that measure $\epsilon_{T}$ has all monotonicity properties [11] relevant to the case of a PCT with just two possible decisions for each pair of objects, i.e., assignment to relation $S$ or to $S^{c}$. Precisely, measure $\epsilon_{T}$ has the following monotonicity properties: $(\mathrm{m} 1)$ - monotonicity w.r.t. the set of criteria, $(\mathrm{m} 2)$ - monotonicity w.r.t. relation $T$, and $(\mathrm{m} 4)-$ monotonicity w.r.t. dominance relation $D_{2}$ over $B$. Definitions of these properties, for the case of a cost-type consistency measure, can be found in the Appendix (Definitions: 13, 14, 15).

Using definitions (14) and (15), one can define v-c upper approximations and v-c boundaries of sets $S$ and $S^{c}$ as in [11].

We define positive regions of relations $S$ and $S^{c}$ as follows:

$$
\begin{aligned}
\operatorname{POS}(S) & =\bigcup_{(a, b) \in \underline{S}} D_{2}^{+}(a, b), \\
\operatorname{POS}\left(S^{c}\right) & =\bigcup_{(a, b) \in \underline{S^{c}}} D_{2}^{-}(a, b) .
\end{aligned}
$$

Positive regions defined above contain pairs of objects sufficiently consistent, i.e., belonging to lower approximations of relation $S(14)$ or $S^{c}(15)$, and can also contain some inconsistent pairs of objects which fall into dominance cones $D_{2}^{+}(\cdot, \cdot)$ or $D_{2}^{-}(\cdot, \cdot)$ originating in pairs of objects from lower approximations of relation $S$ or $S^{c}$, respectively. Moreover, one can define boundary and negative regions of relations $S$ and $S^{c}$ analogously to $[9,13]$. It is also possible to perform further DRSA analysis by calculating the quality of approximation, reducts, and the core $[45,46,48,84,85]$.

\section{Induction of Decision Rules}

After structuring decision examples supplied by the DM into lower approximations of comprehensive preference relations, we induce a generalized description of sufficiently consistent pairs of objects from $S_{P C T}$ in terms of a set of minimal decision rules. An induced set of rules is considered to be a preference model of the DM who gave the pairwise comparisons of reference objects. Each rule is a statement of the type:

$$
\text { if } \Phi \text {, then } \Psi
$$

where $\Phi$ and $\Psi$ denote condition and decision part of the rule, called also premise and conclusion, respectively. The condition part of the rule is a conjunction of elementary conditions concerning individual criteria, and the decision part of the rule suggests assignment of pairs of objects covered by the rule to outranking relation $S$ or to non-outranking relation $S^{c}$. The rule is said to cover a pair of objects $(a, b) \in A \times A$ if this pair satisfies all the elementary conditions of the rule. A pair of objects $(a, b) \in B$ is said to support the rule if this pair satisfies all the elementary conditions and the conclusion of the rule. Rule $r_{T}$, suggesting assignment of covered pairs of objects to relation $T$, is called minimal if there is no other rule $r_{T}^{\prime}$ having premise at least as general as that of $r_{T}$ (i.e., employing subset of elementary conditions of $r_{T}$ and/or more general elementary conditions than $r_{T}$ ) and consistency not worse than that of $r_{T}$ (where by consistency of rule $r_{T}$ we understand the value of a rule consistency measure defined later in this section). In the following, a minimal decision rule is denoted by m-rule. The interest in minimal decision rules comes, obviously, from the fact that they generalize decision examples better than non-minimal rules. Thus, generation of minimal decision rules may be seen as a way to avoid overfitting.

Decision rules are induced so as to cover pairs of objects from lower approximations (14) and (15). However, in some cases it is impossible for a rule to cover only pairs of objects from a lower approximation. 
To handle these cases, the positive region of the considered comprehensive preference relation is computed according to (16) or (17).

Set $\underline{T}$ of pairs of objects belonging to the lower approximation of comprehensive preference relation $T$ is the basis for induction of a set of minimal decision rules that suggest assignment to T. A rule from this set is supported by at least one pair of objects from $\underline{T}$, and it covers pair(s) of objects from $P O S(T)$. The elementary conditions (selectors) that form this rule are built using only evaluations of objects present in the pairs of objects that belong to $\underline{T}$.

Below, we define the syntax of decision rules that generalize description of sufficiently consistent pairs of objects present in a PCT:

$$
\begin{aligned}
& \text { if }\left(\Delta_{i_{1}}(a, b) \geq \delta_{i_{1}}\right) \wedge \ldots \wedge\left(\Delta_{i_{p}}(a, b) \geq \delta_{i_{p}}\right) \wedge \\
&\left(g_{i_{p+1}}(a) \geq r_{i_{p+1}} \wedge g_{i_{p+1}}(b) \leq s_{i_{p+1}}\right) \wedge \ldots \wedge\left(g_{i_{z}}(a) \geq r_{i_{z}} \wedge g_{i_{z}}(b) \leq s_{i_{z}}\right), \\
& \text { then } a S b, \\
& \text { if }\left(\Delta_{i_{1}}(a, b) \leq \delta_{i_{1}}\right) \wedge \ldots \wedge\left(\Delta_{i_{p}}(a, b) \leq \delta_{i_{p}}\right) \wedge \\
&\left(g_{i_{p+1}}(a) \leq r_{i_{p+1}} \wedge g_{i_{p+1}}(b) \geq s_{i_{p+1}}\right) \wedge \ldots \wedge\left(g_{i_{z}}(a) \leq r_{i_{z}} \wedge g_{i_{z}}(b) \geq s_{i_{z}}\right), \\
& \text { then } a S^{c} b,
\end{aligned}
$$

where: $\Delta_{i_{j}}(a, b)$ denotes $g_{i_{j}}(a)-g_{i_{j}}(b), \delta_{i_{j}} \in\left\{g_{i_{j}}(c)-g_{i_{j}}(d):(c, d) \in B\right\}$, for $i_{j} \in\left\{i_{1}, \ldots, i_{p}\right\} \subseteq \mathcal{I}_{G^{N}}$; $\left(r_{i_{j}}, s_{i_{j}}\right) \in\left\{\left(g_{i_{j}}(c), g_{i_{j}}(d)\right):(c, d) \in B\right\}$, for $i_{j} \in\left\{i_{p+1}, \ldots, i_{z}\right\} \subseteq \mathcal{I}_{G^{O}}$. For instance, considering ranking of cars, a decision rule could be "if car $a$ has maximum speed at least $25 \mathrm{~km} / \mathrm{h}$ greater than car $b$ (cardinal criterion) and car $a$ has comfort at least 3 while car $b$ has comfort at most 2 (ordinal criterion), then car $a$ is at least as good as car b", where values 2 and 3 code ordinal evaluations medium and good, respectively.

The rules with syntax (18) are called at least rules, while the rules with syntax (19) are called at most rules. Let us observe that the above syntax of decision rules is concordant with the definition of dominance relation $D_{2}$ over $B$ in the sense that the premise of each decision rule is a positive or negative dominance cone in the $Q$-evaluation space. Moreover, as we work with variable-consistency lower approximations, in order to cover by rules all pairs of objects from $\underline{S}$ and $\underline{S^{c}}$, we have to agree that not all the rules will be fully consistent. For example, it is inevitable that a rule suggesting assignment to relation $S$ covers pairs of objects that do not belong to $S$ but dominate in the $Q$-evaluation space at least one pair of objects from $\underline{S}$ that supports the considered rule. Therefore, in the following, we speak about probabilistic decision rules to underline the fact that not all pairs of objects from $S_{P C T}$ that are covered by a rule have to support this rule.

Decision rules can be characterized by many attractiveness measures (see Greco et al. [55, 59] for a study of some properties of these measures).

Since we work with probabilistic decision rules, it is important to control consistency of these rules. To this end, we define a cost-type rule consistency measure $[12,13]$ denoted by $\widehat{\epsilon}_{T}$. This measure is a function $\widehat{\epsilon}_{T}: R_{T} \rightarrow[0,1]$, where $R_{T}$ is the set of rules suggesting assignment to relation $T$. Let us denote by $\Phi\left(r_{T}\right)$, $\Psi\left(r_{T}\right)$, and $\left\|\Phi\left(r_{T}\right)\right\|$, condition part of rule $r_{T}$, its decision part, and the set of pairs of objects covered by the rule, respectively. Then, measure $\widehat{\epsilon}_{T}$ is defined as:

$$
\widehat{\epsilon}_{T}\left(r_{T}\right)=\frac{\left|\left\|\Phi\left(r_{T}\right)\right\| \cap \neg T\right|}{|\neg T|},
$$

where $\neg T=B \backslash T$ is the complement of relation $T$ w.r.t. set $B$ (obviously, $\neg S=S^{c}$ and $\neg S^{c}=S$ ).

Induced rules have to satisfy the same constraints on consistency as pairs of objects from the lower approximation which serves as a base for rule induction. In particular, each rule $r_{T}$ is required to satisfy threshold $\theta_{T}$, i.e., $\widehat{\epsilon}_{T}\left(r_{T}\right)$ has to be not greater than $\theta_{T}$. In the following, rule $r_{T}$ satisfying threshold $\theta_{T}$ is called sufficiently consistent and denoted by sc-rule. Since rule consistency measure $\widehat{\epsilon}_{T}$ is a counterpart of consistency measure $\epsilon_{T}$ defined as (12) and (13), it can be shown that $\widehat{\epsilon}_{T}$ derives monotonicity properties from $\epsilon_{T}$.

Let us now remind some useful definitions concerning probabilistic decision rules, introduced in [13]. 
A probabilistic decision rule $r_{T}$ suggesting assignment to relation $T$ is discriminant if it covers only pairs of objects belonging to positive region $\operatorname{POS}(T)$. In the following, a discriminant decision rule is denoted by $d$-rule. Moreover, rule $r_{T}$ is robust if there exists a pair of objects $(a, b) \in \underline{T}$ which is a base of $r_{T}$. Considering for example definition (18), it means that $q_{i_{1}}(a, b)=\delta_{i_{1}} \wedge \ldots \wedge q_{i_{p}}(a, b)=\delta_{i_{p}} \wedge q_{i_{p+1}}(a, b)=$ $\left(r_{i_{p+1}}, s_{i_{p+1}}\right) \wedge \ldots \wedge q_{i_{z}}(a, b)=\left(r_{i_{z}}, s_{i_{z}}\right)$. In the following, a robust decision rule is denoted by $r$-rule. Set $R_{T}$ of rules suggesting assignment to relation $T$ is minimal if each pair of objects $(a, b) \in \underline{T}$ is covered by at least one rule $r_{T} \in R_{T}$ and elimination of any rule from $R_{T}$ makes that not all pairs of objects $(a, b) \in \underline{T}$ are covered by the remaining rules. In the following, a minimal set of decision rules is denoted by $m$-set of rules.

Induction of decision rules is a complex problem and many algorithms have been introduced to deal with it. Examples of rule induction algorithms that were defined for DRSA are: by Greco et al. [57], by Dembczyński et al. [29], and by Błaszczyński et al. [12, 13]. In general, rule induction algorithms can be divided into three categories that reflect different induction strategies:

$(\alpha)$ generation of a minimal set of decision rules,

( $\beta$ ) generation of an exhaustive set of decision rules,

$(\gamma)$ generation of a satisfactory set of decision rules.

When applied to a PCT, algorithms from category $(\alpha)$ focus on describing all pairs of objects from lower approximations of $S$ and $S^{c}$ by an m-set of m-rules. Algorithms from category $(\beta)$ generate all m-rules. Category $(\gamma)$ includes algorithms that generate all m-rules that satisfy some a priori defined requirements (concerning, e.g., maximum rule length or minimum support).

In this paper, we apply VC-DomLEM algorithm $[12,13]$ which belongs to category $\alpha$ ). Each of the sets $R_{S}$ and $R_{S^{c}}$ of decision rules induced by VC-DomLEM for comprehensive preference relation $S$ and $S^{c}$, respectively, is an $\mathrm{m}$-set of $\mathrm{m}$-sc-rules (i.e., is a minimal set composed of minimal and sufficiently consistent decision rules). Moreover, we parameterize the algorithm in such a way, that it induces d-rules (technically, this is achieved by choosing covering option $s=1$, which means that each induced rule $r_{T}$ is allowed to cover only pairs of objects belonging to set $P O S(T)$ ). It is important to note that the rules generated by VC-DomLEM do not have to be robust, which means that each rule $r_{T}$ can employ elementary conditions created using evaluations in $Q$-evaluation space of different pairs of objects from $\underline{\mathrm{T}}$.

\section{Application of Decision Rules}

After induction of decision rules, the next step of the proposed methodology to multicriteria ranking is the application of induced rules on set $A$. This application yields a fuzzy preference structure on set $A$. Each pair of objects $(a, b) \in A \times A$ can be covered by some decision rules suggesting assignment to relation $S$ and/or to relation $S^{c}$. It can be also not covered by any rule. In order to represent each of these situations, first we treat each rule $r_{T}$ covering pair $(a, b)$ as an argument (piece of evidence) for assignment of this pair to relation $T$. Second, we take into account strength $\sigma$ of each argument (rule $r_{T}$ ) defined in the following way:

$$
\sigma\left(r_{T}\right)=\left(1-\widehat{\epsilon}_{T}\left(r_{T}\right)\right) c f\left(r_{T}\right)
$$

where $c f\left(r_{T}\right)$ denotes coverage factor of rule $r_{T}$, defined as the ratio of the number of pairs of objects supporting $r_{T}$ and the cardinality of relation $T$. In this way, the higher the consistency of the rule (i.e., the lower the value of $\widehat{\epsilon}_{T}$ ), and the greater the number of pairs of objects supporting the rule, the stronger the argument for assignment of pair $(a, b)$ to relation $T$. Third, analogously to [35, 36], we accumulate the strength of the arguments supporting assignment of pair $(a, b)$ to relation $T$ by taking maximum strength of these arguments. This way, performing all three steps for every pair of objects $(a, b) \in A \times A$, we get two 
fuzzy preference relations over $A$, denoted by $\mathcal{S}$ and $\mathcal{S}^{c}$, defined as:

$$
\begin{aligned}
\mathcal{S}(a, b) & =\left\{\begin{array}{l}
\max \left\{\sigma\left(r_{S}\right): r_{S} \in R_{S} \text { and } r_{S} \text { covers }(a, b)\right\}, \text { if } a \neq b \\
1, \text { if } a=b
\end{array}\right. \\
\mathcal{S}^{c}(a, b) & =\left\{\begin{array}{l}
\max \left\{\sigma\left(r_{S^{c}}\right): r_{S^{c}} \in R_{S^{c}} \text { and } r_{S^{c}} \text { covers }(a, b)\right\}, \text { if } a \neq b \\
0, \text { if } a=b
\end{array}\right.
\end{aligned}
$$

Let us observe that relation $\mathcal{S}$ is reflexive and relation $\mathcal{S}^{c}$ is irreflexive.

The fuzzy preference structure on $A$, composed of $\mathcal{S}$ and $\mathcal{S}^{c}$, can be represented by a preference graph. It is a directed weighted multigraph $\mathcal{G}$. Each vertex (node) $v_{a}$ of the preference graph corresponds to exactly one object $a \in A$. One can distinguish in $\mathcal{G}$ two types of arcs: $\mathcal{S}$-arcs and $\mathcal{S}^{c}$-arcs. Each $\mathcal{S}$-arc between vertices $v_{a}$ and $v_{b}$ is weighted by value $\mathcal{S}(a, b)$. Analogously, each $\mathcal{S}^{c}$-arc between vertices $v_{a}$ and $v_{b}$ is weighted by value $\mathcal{S}^{c}(a, b) . \mathcal{G}$ is a multigraph since there may be one $\mathcal{S}$-arc and one $\mathcal{S}^{c}$-arc for each pair of objects $(a, b) \in A \times A$. A final recommendation for the multicriteria ranking problem at hand, in terms of a complete or partial preorder of all objects belonging to set $A$, can be obtained upon a suitable exploitation of the preference graph.

\section{Exploitation of Preference Graph}

\subsection{Representation of fuzzy relations $\mathcal{S}$ and $\mathcal{S}^{c}$ by a preference graph}

The exploitation of preference graph $\mathcal{G}$ resulting from application of induced decision rules on set $A$ is not an easy task, especially because this graph represents two fuzzy relations $\mathcal{S}$ and $\mathcal{S}^{c}$. This task is more complex than the exploitation of a preference graph representing just one fuzzy relation, well studied in the literature $[4,14-17,20-22,30,71,73]$.

Preference graphs representing only one fuzzy relation are obtained, e.g., in several decision support methods proposed in the field of MCDA, in which preferences of a DM are modeled in terms of binary relations. Among these methods, one can mention ELECTRE III $[33,75]$ and PROMETHEE I and II $[23,24]$. When preferences are modeled in terms of binary relations, the key question is the existence of evidence in favor of the considered relation. For example, in case of outranking relation $S$ concerned in the methods from ELECTRE family, the evidence concerns the sentence $a S b$ and/or $b S a$, for any pair of objects $a, b \in A$. In reality, the evidence is never complete, thus inducing a graded (fuzzy) relation $a S b$, i.e., " $a$ is at least as good as $b$, up to a certain degree of certainty".

It is reasonable to claim that considering only evidence in favor of the considered binary relation does not allow to catch the reality of some decision problems. In fact, such an approach leads to a situation where the evidence in disfavor of a sentence is semantically considered - and thus modeled - as the evidence in favor of the opposite sentence. This mental restriction may induce not only misunderstandings but, which is even more important, it may also imply some loss of information (a good example clarifying this point, concerning government composition, is presented by Fortemps and Słowiński [36]). Therefore, in this paper, given a pair of objects $(a, b) \in A \times A$, we consider not only the decision rules supporting conclusion $a S b$, but also the rules supporting the opposite conclusion, i.e., conclusion $a S^{c} b$. In this way, we take into account the arguments in favor of preference of $a$ over $b$ and in disfavor of it; in the following, they will be also called positive and negative arguments, respectively. As described in Section 7, the accumulated strength of the positive arguments is reflected by the value $\mathcal{S}(a, b)$, while the accumulated strength of the negative arguments is reflected by the value $\mathcal{S}^{c}(a, b)$.

The information contained in a preference graph representing two fuzzy preference relations $\mathcal{S}$ and $\mathcal{S}^{c}$ over set $A$ can be also expressed in the form of four graded (fuzzy) outranking relations $\mathcal{S}^{\mathcal{T}}, \mathcal{S}^{\mathcal{F}}, \mathcal{S}^{\mathcal{U}}, \mathcal{S}^{\mathcal{K}} \in \mathbf{R}_{A}$, considered, e.g., by Tsoukias and Vincke [89], Perny and Tsoukias [72], Fortemps and Słowiński [36]. These binary relations correspond to four truth values of Belnap [5,6]: $T$ (true), $F$ (false), $U$ (unknown), and $K$ 
(contradictory). Relations $\mathcal{S}^{\mathcal{T}}, \mathcal{S}^{\mathcal{F}}, \mathcal{S}^{\mathcal{U}}$, and $\mathcal{S}^{\mathcal{K}}$, are defined in the following way:

$$
\begin{aligned}
& \mathcal{S}^{\mathcal{T}}(a, b)=\min \left(\mathcal{S}(a, b), 1-\mathcal{S}^{c}(a, b)\right), \\
& \mathcal{S}^{\mathcal{F}}(a, b)=\min \left(1-\mathcal{S}(a, b), \mathcal{S}^{c}(a, b)\right), \\
& \mathcal{S}^{\mathcal{K}}(a, b)=\min \left(\mathcal{S}(a, b), \mathcal{S}^{c}(a, b)\right), \\
& \mathcal{S}^{\mathcal{U}}(a, b)=\min \left(1-\mathcal{S}(a, b), 1-\mathcal{S}^{c}(a, b)\right),
\end{aligned}
$$

where $a, b \in A$. Is is worth noting that the above definitions relate to preference, indifference, and incomparability indexes introduced by Bisdorff [7].

\subsection{Review of possible exploitation procedures}

Given a preference graph $\mathcal{G}$, one can propose several exploitation techniques that lead to final recommendation for the multicriteria ranking problem at hand, in terms of a complete or partial preorder of all objects from set $A$. We distinguish the following approaches:

(i) direct exploitation of fuzzy preference relations $\mathcal{S}$ and $\mathcal{S}^{c}$ by the Fuzzy Net Flow Score (FNFS) procedure [35],

(ii) exploitation of the four graded outranking relations $\mathcal{S}^{\mathcal{T}}, \mathcal{S}^{\mathcal{F}}, \mathcal{S}^{\mathcal{U}}$, and $\mathcal{S}^{\mathcal{K}}$ in the way proposed by Fortemps and Słowiński [36],

(iii) independent exploitation of fuzzy preference relations $\mathcal{S}$ and $\mathcal{S}^{c}$,

(iv) suitable transformation of preference graph $\mathcal{G}$ to another graph $\mathcal{G}^{\prime}$ representing only one fuzzy relation, then exploitation of this relation leading to complete or partial preorder over $A$.

Approach $(i)$ is based on scoring function $S^{N F}: A \rightarrow \Re$ defined as:

$$
S^{N F}(a)=\sum_{b \in A \backslash\{a\}} \mathcal{S}(a, b)-\mathcal{S}(b, a)-\mathcal{S}^{c}(a, b)+\mathcal{S}^{c}(b, a) .
$$

Function $S^{N F}$ induces a weak order over $A$, which is a solution of the considered multicriteria ranking problem. Note that this scoring function was also considered by Greco et al. [53], in case of crisp relations $\mathcal{S}$ and $\mathcal{S}^{c}$. In approach $(i i)$, one associates with each object $a \in A$ a vector $\bar{a}$, defined as:

$$
\bar{a}=\left(t_{b}, t_{c}, \ldots, f_{b}, f_{c}, \ldots\right),
$$

where $t_{b}$ (respectively, $f_{b}$ ) denotes $\mathcal{S}^{\mathcal{T}}(a, b)$ (respectively, $\mathcal{S}^{\mathcal{F}}(b, a)$ ). Then, all vectors corresponding to objects from set $A$ are sorted in the non-decreasing order and compared lexicographically (to resolve ties, one can also take into account for each object $a \in A$ additional vectors composed of values $\mathcal{S}^{\mathcal{K}}(a, \cdot)$ or $\left.\mathcal{S}^{\mathcal{U}}(a, \cdot)\right)$. Such leximin-scoring procedure yields a partial preorder over $A$. The idea of approach (iii) is to exploit relations $\mathcal{S}$ and $\mathcal{S}^{c}$ independently, obtaining two separate preorders (complete or partial), and then to conjunct these preorders in the same way as in the ELECTRE III method [33, 75]. This leads to obtaining a partial preorder over $A$.

\subsection{Fusion of fuzzy relations $\mathcal{S}$ and $\mathcal{S}^{c}$ in order to exploit a single relation}

In the following, we will concentrate on approach $(i v)$, mainly for three reasons. The first one is that the exploitation of a fuzzy relation over a set of objects is well studied in the literature, as already mentioned earlier in this section. Many ranking methods have been proposed in this subject. The diversity of ranking methods calls for a systematic comparison of their formal properties, which is, however, missing. The second reason for concentrating on approach $(i v)$ is that, using a suitable transformation of preference graph $\mathcal{G}$ and an appropriate ranking method to exploit the transformed graph $\mathcal{G}^{\prime}$, it is possible to obtain the same final ranking as in approach $(i)$. Thus, approach $(i v)$ can be seen as a framework that encompasses approach $(i)$. 
The third reason is that when applied to set $A$, most of the ranking methods considered in the literature yield a weak order over $A$, which is generally acknowledged to be more operational for the DM than a partial preorder that can be obtained in approaches $(i i)$ and $($ iii $)$.

The suitable transformation of preference graph $\mathcal{G}$ representing two fuzzy preference relations $\mathcal{S}$ and $\mathcal{S}^{c}$ to graph $\mathcal{G}^{\prime}$ representing one fuzzy relation $\mathcal{R} \in \mathbf{R}_{A}$ consists in defining relation $\mathcal{R}$ in the following way:

$$
\mathcal{R}(a, b)=\frac{\mathcal{S}(a, b)+\left(1-\mathcal{S}^{c}(a, b)\right)}{2},
$$

where $a, b \in A$. Let us observe that scoring function $S^{N F}$ defined by (28) can be expressed in terms of $\mathcal{R}$ as: $S^{N F}(a)=2\left[\sum_{b \in A \backslash\{a\}} \mathcal{R}(a, b)-\mathcal{R}(b, a)\right]$. Moreover, relation $\mathcal{R}$ is reflexive, i.e., $\mathcal{R}(a, a)=1$ for all $a \in A$. In the following, considering exploitation of relation $\mathcal{R}$, we assume that this relation has no "structural properties" [18], i.e., we assume (what seems to be the case) that $\mathcal{R}$ may be any fuzzy relation over $A$. The rationale for this assumption is that relation $\mathcal{R}$ depends only on a considered set of decision rules, and, in general, this set of rules does not depend on $A$.

\subsection{Review of ranking methods}

In the literature, one can find many ranking methods "dedicated" to exploitation of a fuzzy relation over a set of objects [14, 16, 20-22, 73]. On the other hand, as argued by Arrow and Raynaud [3], one can be also interested in another approach to rank objects which consists in (downward) iterative application of a choice function. Let us denote by $\mathcal{P}_{A}$ the set of all nonempty subsets of a finite set of objects $A$. Then, choice function $c f$ is a function

$$
\text { cf }: \mathcal{P}_{A} \times \mathbf{R}_{A} \rightarrow \mathcal{P}_{A} .
$$

A choice function associates with each nonempty set $A^{\prime} \subseteq A$ and each fuzzy relation $R$ over $A$, a nonempty choice set $\operatorname{cf}\left(A^{\prime}, R\right) \subseteq A^{\prime}$, which may be interpreted as the set of the "best" objects in $A^{\prime}$ given relation $R$. Iterative application of a choice function on a finite set $A$ was considered, e.g., in [19, 21, 22]. It leads to obtaining a weak order over $A$. Let us denote by $A^{i} \subseteq A$ the set of objects considered in $i$-th iteration and by $|A|$ the cardinality of set $A$. Obviously, $A^{1}=A$. In $i$-th iteration, $i \in\{1,2, \ldots,|A|\}$, given choice function $c f$ is applied to set $A^{i}$. Then, the objects belonging to choice set $c f\left(A^{i}, R\right)$ are put in $i$-th rank of the constructed ranking and removed from set $A^{i}$. Thus, $A^{i+1}=A^{i} \backslash c f\left(A^{i}, R\right)$. The construction of a final ranking is finished when this ranking contains all objects from set $A$.

Most of the proposed "dedicated" ranking methods as well as ranking methods based on iterative application of a choice function employ a scoring function. Given a finite set of objects $A$ and a fuzzy relation $R$ over $A$, scoring function is used to evaluate relative performance of each object $a \in A$ w.r.t. the objects in nonempty set $A^{\prime} \subseteq A$, taking into account relation $R$. Thus, scoring function $s f$ is a function

$$
\text { sf }: A \times \mathcal{P}_{A} \times \mathbf{R}_{A} \rightarrow \Re .
$$

Value $s f\left(a, A^{\prime}, R\right)$ denotes the score of object $a \in A$ calculated w.r.t. the objects in $A^{\prime} \subseteq A$, given fuzzy relation $R$.

Let define two generic score-based ranking methods: single-stage ranking method $\left(\succeq^{1}\right)$ and multi-stage ranking method $\left(\succeq^{i}\right)$. These ranking methods are parameterized by a set of objects $A$, a fuzzy relation $R$ over $A$, and a scoring function $s f$. Moreover, they yield a weak order over $A$ :

- $\succeq^{1}(A, R, s f)$ :

assign score $s f(a, A, R)$ to each object $a \in A$ and rank all the objects from set $A$ according to their scores, in such a way that the higher the score of an object, the lower its rank (objects with the same score belong to the same rank);

- $\succeq^{i}(A, R, s f)$ :

(1) define choice function $c f$ as: $c f\left(A^{\prime}, R\right)=\left\{a \in A^{\prime}: \operatorname{sf}\left(a, A^{\prime}, R\right) \geq s f\left(b, A^{\prime}, R\right)\right.$ for all $\left.b \in A^{\prime}\right\}$, where $A^{\prime} \subseteq A$, i.e., in such a way that it chooses subset of $A^{\prime}$ composed of objects with the highest score; 
(2) perform (downward) iterative application of choice function $c f$ on set $A$.

Clearly, the aforementioned "dedicated" ranking methods are instances of $\succeq$ ', differing only by the definition of function $s f$. Analogously, ranking methods based on iterative choice considered by Bouyssou and Pirlot [21], and Bouyssou and Vincke [22] are instances of $\succeq^{i}$, differing only by the definition of function $s f$.

Let us consider a finite set of objects $A$ and a fuzzy relation $R$ over $A$. Then, according to Barrett et al. [4], the score of any object $a \in A$ w.r.t. the objects in any set $A^{\prime} \subseteq A$ can be calculated using one of the following scoring functions:

$$
\begin{aligned}
& \text { max in favor: } \quad \operatorname{MF}\left(a, A^{\prime}, R\right) \quad=\max _{b \in A^{\prime} \backslash\{a\}} R(a, b) \text {, } \\
& \text { min in favor: } \quad m F\left(a, A^{\prime}, R\right) \quad=\min _{b \in A^{\prime} \backslash\{a\}} R(a, b) \text {, } \\
& \text { sum in favor: } \quad S F\left(a, A^{\prime}, R\right) \quad=\sum_{b \in A^{\prime} \backslash\{a\}} R(a, b) \text {, } \\
& -\max \text { against }: \quad-M A\left(a, A^{\prime}, R\right) \quad=\quad-\max _{b \in A^{\prime} \backslash\{a\}} R(b, a), \\
& - \text { min against : } \quad-m A\left(a, A^{\prime}, R\right) \quad-\min _{b \in A^{\prime} \backslash\{a\}} R(b, a), \\
& \text {-sum against : } \quad-S A\left(a, A^{\prime}, R\right) \quad-\sum_{b \in A^{\prime} \backslash\{a\}} R(b, a), \\
& \text { max difference : } \quad M D\left(a, A^{\prime}, R\right) \quad=\quad \max _{b \in A^{\prime} \backslash\{a\}} R(a, b)-R(b, a) \text {, } \\
& \text { min difference : } \quad m D\left(a, A^{\prime}, R\right) \quad=\min _{b \in A^{\prime} \backslash\{a\}} R(a, b)-R(b, a) \text {, } \\
& \text { sum of differences : } \quad S D\left(a, A^{\prime}, R\right) \quad=\sum_{b \in A^{\prime} \backslash\{a\}} R(a, b)-R(b, a) \text {. }
\end{aligned}
$$

It is worth noting that $S D\left(a, A^{\prime}, R\right)$ is a sum of $S F\left(a, A^{\prime}, R\right)$ and $-S A\left(a, A^{\prime}, R\right)$.

Given a finite set of objects $A$ and a fuzzy relation $R$ over $A$, we consider exploitation of relation $R$ using one of the following ranking methods, well studied in the literature:

(1) Net Flow Rule [16, 22], defined as:

$$
N F R(A, R)=\succeq^{1}(A, R, S D)
$$

(2) Iterative Net Flow Rule [22], defined as:

$$
\operatorname{It.NFR}(A, R)=\succeq^{i}(A, R, S D),
$$

(3) Min in Favor [14, 21, 22, 73], defined as:

$$
\operatorname{MiF}(A, R)=\succeq^{1}(A, R, m F)
$$

(4) Iterative Min in Favor [21], defined as:

$$
\operatorname{It.MiF}(A, R)=\succeq^{i}(A, R, m F),
$$

(5) Leaving and Entering Flows [20], defined as:

$$
L / E(A, R)=\succeq^{1}(A, R, S F) \cap \succeq^{1}(A, R,-S A) .
$$


As can be seen, considered ranking methods employ only some of the defined scoring functions, namely: $m F$ (33), $S F(34),-S A(37)$, and $S D(40)$.

$N F R$ orders objects according to their net flow scores. It has a long history in social choice theory $[2,34]$. It coincides with the rule of Copeland [cf. 34, 60, 79] when $R$ is crisp. When $R(a, b)$ is interpreted as a percentage of voters considering that $a$ is preferred or indifferent to $b(a, b \in A)$, it corresponds to the well-known rule of Borda [cf. 34, 91]. Moreover, $N F R$ is used in the PROMETHEE II outranking method [23, 24]. It.NFR consists in iterative application of a choice function that chooses objects with the highest value of scoring function $S D(40)$. This ranking method was originally called the Repeated Net Flow Rule and denoted by $R N F R$ [22]. L/E is used in the PROMETHEE I method [23, 24]. This ranking method allows any two objects $a, b \in A$ to be declared incomparable. This happens when two conclusions concerning ranking of these objects, one conclusion resulting only from the comparison of their leaving flows, i.e., values $S F(\cdot, A, R)$, and the other one resulting only from the comparison of their entering flows, i.e., values $-(-S A(\cdot, A, R))$, are contradictory. Such contradiction occurs, e.g., when $S F(a, A, R)>S F(b, A, R)$, while $-S A(a, A, R)<-S A(b, A, R)$. It should be noticed that $N F R$ and $L / E$ make use of the "cardinal" properties of values $R(a, b)$, with $a, b \in A$. On the other hand, MiF represents a prudent approach as it is purely "ordinal" - it uses values $R(a, b)$ as if they were a numerical representation of a credibility of a crisp relation between $a$ and $b$. Thus, from the fact that $R(a, b) \geq R(c, d)$ it concludes only that the relation between $a$ and $b$ is not less credible than the relation between $c$ and $d$, with $a, b, c, d \in A$.

Now, let us come back and explain the sentence "approach $(i v)$ can be seen as a framework that encompasses approach $(i)$ ", which appeared in the context of the four approaches for exploitation of preference graph $\mathcal{G}$. By saying this we meant that the weak order over $A$ obtained using formula $(28)$ is the same as the weak order over $A$ obtained using $\operatorname{NFR}(A, \mathcal{R})$.

\subsection{Analysis of desirable properties of the ranking methods}

In the literature, one can find many properties considered in the context of ranking methods exploiting fuzzy relations. These properties concern the result of application of a ranking method to any fuzzy relation or to a fuzzy relation with particular features, e.g., a relation which is crisp and transitive. It should be noticed, however, that these properties concern only the dependencies between the exploited fuzzy relation and obtained final ranking. Thus, they do not concern the dependencies between comprehensive preference relations $S, S^{c}$ and the final ranking.

The properties of ranking methods can be basically divided into two non-disjoint groups [14, 22]: desirable properties and "characterizing" properties. The former reflect some expectations of a DM w.r.t. the final ranking produced by a ranking method. The latter reflect intrinsic characteristics of a ranking method; given a ranking method, the research concerning "characterizing" properties aims at defining minimal sets of properties that a given ranking method is the only one to satisfy $[14,16,20,22,73]$. Since our goal is to obtain the "best" ranking, we compare different ranking methods w.r.t. desirable properties only. The same way was adopted, e.g., by Vincke [90], in the context of exploitation of a crisp relation.

In general, different properties can be considered desirable in different decision problems (see Bouyssou and Vincke [22]). We propose a list of properties that seem to be of interest for most decision problems. Moreover, in order to avoid a situation where all considered ranking methods become incomparable (nondominated), we suppose a priority order of considered desirable properties (which, from our point of view, reflects relative importance of these properties). This order is to be used only to resolve situations where two or more ranking methods satisfy the same maximum number of properties.

We find it reasonable to consider the following ordered list of desirable properties of a ranking method to be applied to exploitation of fuzzy relation $\mathcal{R}(29)$ :

(1) Neutrality (property $N$ )

This property was considered, e.g., by Bouyssou [14, 16], Bouyssou and Perny [20], Bouyssou and Vincke [22], and by Pirlot [73].

Definition 1 (Neutrality). A ranking method $\succeq$ is neutral if, for any finite set of objects $A$ and any fuzzy relation $R$ over $A$ : 
$(\sigma$ is a permutation on $A) \Rightarrow\left(a \succeq(A, R) b \Leftrightarrow \sigma(a) \succeq\left(A, R^{\sigma}\right) \sigma(b)\right.$, for all $\left.a, b \in A\right)$, where $R^{\sigma}$ is defined by $R^{\sigma}(\sigma(a), \sigma(b))=R(a, b)$, for all $a, b \in A$.

Thus, neutrality expresses the fact that a ranking method does not discriminate between objects just because of their labels (or, in other words, their order in the considered set $A$ ). It is a classical property in this context [see, e.g., 60, 79].

(2) Monotonicity (property $M$ )

Property of this name was considered, e.g. by Bouyssou and Perny [20], Pirlot [73], and by Bouyssou and Vincke [22], although the proposed definitions were semantically slightly different. In this paper, we adopt the definition of monotonicity property given by Bouyssou and Perny [20]. Intuitively, monotonicity says that improving an object cannot decrease its position in the ranking and, moreover, deteriorating an object cannot improve its position in the ranking. In our opinion, the other two definitions previously considered miss at least one aspect of this intuitive formulation. Thus, we propose the following formulation of the monotonicity property.

Definition 2 (Monotonicity). A ranking method $\succeq$ is monotonic if, for any finite set of objects $A$, any fuzzy relation $R$ over $A$, and any $a, b \in A$ :

$\left(a \succeq(A, R) b \Rightarrow a \succeq\left(A, R^{\prime}\right) b\right)$,

where $R^{\prime}$ is identical to $R$ except that

$\left(R^{\prime}(a, c)>R(a, c)\right.$ or $R^{\prime}(c, a)<R(c, a)$, for some $\left.c \in A \backslash\{a\}\right)$ or

$\left(R^{\prime}(b, d)<R(b, d)\right.$ or $R^{\prime}(d, b)>R(d, b)$, for some $\left.d \in A \backslash\{b\}\right)$.

Precisely, the definition given by Pirlot [73] w.r.t. the difference between $R^{\prime}$ and $R$ concerns only that $\left(R^{\prime}(a, c)>R(a, c)\right.$, for some $\left.c \in A \backslash\{a\}\right)$ or

$\left(R^{\prime}(b, d)<R(b, d)\right.$, for some $\left.d \in A \backslash\{b\}\right)$.

Moreover, the definition given by Bouyssou and Vincke [22] lacks the second part of the above disjunction, i.e. the part concerning object $b$ : $\left(R^{\prime}(b, d)<R(b, d)\right.$ or $R^{\prime}(d, b)>R(d, b)$, for some $\left.d \in A \backslash\{b\}\right)$.

(3) Covering Compatibility (property $C C$ )

This property was considered, e.g., by Bouyssou and Vincke [22] and by Vincke [90] (who called it respect for the covering relation).

Definition 3 (Covering Compatibility). A ranking method $\succeq$ is covering compatible if, for any finite set of objects $A$, any fuzzy relation $R$ over $A$, and any $a, b \in A$ :

$(R(a, b) \geq R(b, a)$, and for all $c \in A \backslash\{a, b\}, R(a, c) \geq R(b, c)$ and $R(c, a) \leq R(c, b)) \Rightarrow a \succeq(A, R) b$.

Thus, property $C C$ expresses the intuition that when $a$ "covers" $b, b$ should not be ranked before $a$. Our interest in this property results also from a very important fact - in case of exploitation of fuzzy relation $\mathcal{R}$ defined by (29), property $C C$ of applied ranking method guaranties that the final ranking produced by this method respects dominance relation $D$ over set $A$. Formally, this can be expressed by:

Corollary 1. Given any two objects $a, b \in A$, such that $a D b$, property $C C$ of ranking method $\succeq$ applied to exploitation of relation $\mathcal{R}(29)$ guaranties that $a \succeq(A, \mathcal{R}) b$.

Proof. See the Appendix.

(4) Independence of Non-Discriminating Objects (property INDO)

This property was considered, e.g., by Bouyssou and Vincke [22] (where it was called independence of non-discriminating alternatives) and by Vincke [90] (who called it independence of non-discriminating elements: weak version). 
Definition 4 (Independence of Non-Discriminating Objects). A ranking method $\succeq$ is independent of non-discriminating objects if, for any finite set of objects $A$ and any fuzzy relation $R$ over $A$ : $\left(R(a, b)=k\right.$ and $R(b, a)=k^{\prime}$, for all $a \in A^{\prime}$ and all $b \in A \backslash A^{\prime}$, with $\left.A^{\prime} \subset A\right) \Rightarrow\left(\succeq\left(A^{\prime}, R / A^{\prime}\right)=\succeq\right.$ $\left.(A, R) / A^{\prime}\right)$.

In the above definition, set $A \backslash A^{\prime}$ is composed of non-discriminating objects. Thus, independence of non-discriminating objects says that when there is a subset of objects that compare in the same way to all other objects, the ranking of the other objects is not affected by the presence of this subset.

(5) Independence of Circuits (property IC)

This property was considered, e.g., by Bouyssou [16] and by Bouyssou and Vincke [22]. It reflects the way in which a ranking method deals with circuits (cycles) in the considered fuzzy relation. It uses the concept of circuit equivalency of two fuzzy relations.

Definition 5 (Circuit Equivalency). Let us consider a finite set of objects $A$. Two fuzzy relations $R$ and $R^{\prime}$ over $A$ are circuit-equivalent if $R^{\prime}$ is identical to $R$ except that, for some distinct $a, b, c \in A$ and some $\epsilon \in[-1,1]$ :

$\left(R^{\prime}(a, b)=R(a, b)+\epsilon\right.$ and $\left.R^{\prime}(b, a)=R(b, a)+\epsilon\right)$ or
$\left(R^{\prime}(a, b)=R(a, b)+\epsilon, R^{\prime}(b, c)=R(b, c)+\epsilon\right.$ and $\left.R^{\prime}(c, a)=R(c, a)+\epsilon\right)$.

Thus, $R^{\prime}$ and $R$ are circuit-equivalent if they are identical except for a circuit of length 2 or 3 on which a positive or negative value has been added.

Definition 6 (Independence of Circuits). A ranking method $\succeq$ is independent of circuits if, for any finite set of objects $A$ and any two fuzzy relations $R$ and $R^{\prime}$ over $A$ :

$\left(R^{\prime}\right.$ and $R$ are circuit-equivalent $) \Rightarrow\left(\succeq\left(A, R^{\prime}\right)=\succeq(A, R)\right)$.

According to Bouyssou and Vincke [22], property $I C$ has a straightforward interpretation. When $R^{\prime}$ and $R$ are circuit-equivalent via a circuit of length 2, independence of circuits implies that the ranking is only influenced by the differences $R(a, b)-R(b, a)$. When $R^{\prime}$ and $R$ are circuit-equivalent via a circuit of length 3, independence of circuits implies that intransitivities of the kind $R(a, b)>0, R(b, c)>0$ and $R(c, a)>0$ can be "wiped out". It is important to notice that property $I C$ makes an explicit use of the "cardinal" properties of values $R(a, b)$, with $a, b \in A$ (except for the particular case in which both $R$ and $R^{\prime}$ are crisp).

(6) Ordinality (property $O$ )

This property was considered, e.g., by Bouyssou [14], Pirlot [73], Bouyssou and Pirlot [21], and by Bouyssou and Vincke [22].

Definition 7 (Ordinality). A ranking method $\succeq$ is ordinal if, for any finite set of objects $A$, any fuzzy relation $R$ over $A$, and any strictly increasing and one-to-one transformation $\phi:[0,1] \rightarrow[0,1]$ : $\succeq(A, \phi[R])=\succeq(A, R)$, where $\phi[R]$ is the fuzzy relation on $A$ such that $\phi[R](a, b)=\phi(R(a, b))$, for all $a, b \in A$.

Thus, ordinality implies that a ranking method should not make use of the "cardinal" properties of values $R(a, b)$, with $a, b \in A$.

(7) Continuity (property $C$ )

This property was considered, e.g., by Bouyssou [14], Bouyssou and Pirlot [21], and by Bouyssou and Vincke [22]. It uses the concept of convergence of a sequence of fuzzy relations to a given fuzzy relation. 
Definition 8 (Convergence). Let us consider a finite set of objects $A$ and a sequence of fuzzy relations $\left(R^{i}, i=1, \ldots\right)$ that are defined over $A$. We say that this sequence converges to fuzzy relation $R$ if for any (arbitrarily small) $\epsilon>0$ there is an integer $k$, such that for all $j>k$ and all $a, b \in A$, we have $\left|R^{j}(a, b)-R(a, b)\right|<\epsilon$.

Definition 9 (Continuity). A ranking method $\succeq$ is continuous if, for any finite set of objects $A$, any fuzzy relation $R$ over $A$, any sequence of fuzzy relations $\left(R^{i}, i=1, \ldots\right)$ converging to $R$, and any $a, b \in A$ :

$\left(a \succeq\left(A, R^{i}\right) b\right.$ for all $R^{i}$ in the sequence $) \Rightarrow(a \succeq(A, R) b)$.

Thus, continuity says that "small" changes in an exploited fuzzy relation should not lead to radical changes in the final ranking produced by a ranking method.

(8) Faithfulness (property $F$ )

This property was considered, e.g., by Bouyssou and Vincke [22] and by Vincke [90] (who called it respect for the data 1.1).

Definition 10 (Faithfulness). A ranking method $\succeq$ is faithful if, for any finite set of objects $A$ and any relation $R$ over $A$ :

$(R$ is a weak order over $A) \Rightarrow(\succeq(A, R)=R)$.

As can be seen, faithfulness concerns behavior of a ranking method in a special case when considered relation $R$ is crisp and, moreover, it is a weak order over $A$. This property says that a ranking method applied to a weak order should preserve it.

(9) Data-Preservation (property DP)

This property was considered, e.g., by Bouyssou and Vincke [22] (where it was called data-preservation 1) and by Vincke [90] (who called it respect for the data 1.3).

Definition 11 (Data-Preservation). A ranking method $\succeq$ is data-preserving if, for any finite set of objects $A$ and any relation $R$ over $A$ :

( $R$ is a transitive crisp relation over $A) \Rightarrow(R \subseteq \succeq(A, R))$.

Thus, data-preservation says that when it is possible to obtain a partial preorder on the basis of $R$ without deleting information contained in this relation, a ranking method should do so. It is important to note that property $D P$ is not implied by property $F$ and vice versa.

(10) Greatest-Faithfulness (property GF)

This property was considered, e.g., by Bouyssou and Pirlot [21], and by Bouyssou and Vincke [22].

Definition 12 (Greatest-Faithfulness). A ranking method $\succeq$ is greatest-faithful if, for any finite set of objects $A$ and any relation $R$ over $A$ :

$(R$ is a crisp relation and $G(A, R) \neq \emptyset) \Rightarrow(G(A, \succeq(A, R)) \subseteq G(A, R))$.

Greatest-faithfulness says that if there are some greatest elements of a given set $A$, then the top-ranked objects should be chosen among them (observe that in case of a ranking method that yields a partial preorder over $A$, there may be no top-ranked objects, i.e., set $G(A, \succeq(A, R))$ may be empty). Let us note, however, that some authors [e.g., 21] do not find greatest-faithfulness as a particularly intuitive requirement for a ranking method, as this property concerns only the first equivalence class of the obtained ranking (they rather consider this property in the context of choice methods). Moreover, in spite of names, it should be noticed that a faithful ranking method is not necessarily greatest-faithful and vice versa. 


\subsection{The choice of the best ranking method to work out a recommendation}

Before verifying properties of the five considered ranking methods, let us make a note concerning reflexivity of an exploited fuzzy relation $R \in \mathbf{R}_{A}$. Vincke [90] and Pirlot [73] assumed $R$ to be irreflexive. Bouyssou [14], Bouyssou and Perny [20] assumed that $R(a, b)$ is defined only for pairs of objects $(a, b) \in A \times A$ such that $a \neq b$. Finally, Bouyssou and Vincke [22], Bouyssou and Pirlot [21] assumed that $R$ is reflexive. In this paper, exploited relation $\mathcal{R}(29)$ is reflexive. However, since each of the five ranking methods analyzed here makes use of a scoring function that for any finite set of objects $A$ and any fuzzy relation $R$ over $A$ does not take into account values $R(a, a)$, with $a \in A$, previous results concerning properties of the five ranking methods hold.

Table 1 presents properties of the five considered ranking methods. In this table, symbols $\mathrm{T}$ and $\mathrm{F}$ denote presence and absence of a given property, respectively. Moreover, bold font is used in case when a given pair (Property, RM) was already considered in the literature (where a proof or a counterexample was given), while italics is used otherwise, in which case a proof or a counterexample is given in the Appendix. Note that in the row corresponding to property $M$, some symbols $\mathrm{T}$ and $\mathrm{F}$ are in italics due to adoption of particular definition of this property (see Definition 2).

\begin{tabular}{cccccc} 
Table 1: Properties of considered ranking methods for exploitation of a fuzzy relation \\
\hline Property $/$ RM & $N F R$ & It.NFR & $M i F$ & It.MiF & $L / E$ \\
\hline$N$ & $\mathbf{T}$ & $\mathbf{T}$ & $\mathbf{T}$ & $T$ & $T$ \\
$M$ & $T$ & $F$ & $T$ & $F$ & $\mathbf{T}$ \\
$C C$ & $\mathbf{T}$ & $\mathbf{T}$ & $T$ & $T$ & $T$ \\
$I N D O$ & $\mathbf{T}$ & $\mathbf{T}$ & $F$ & $F$ & $T$ \\
$I C$ & $\mathbf{T}$ & $F$ & $\mathbf{F}$ & $F$ & $F$ \\
$O$ & $\mathbf{F}$ & $F$ & $\mathbf{T}$ & $\mathbf{T}$ & $F$ \\
$C$ & $\mathbf{T}$ & $F$ & $\mathbf{T}$ & $\mathbf{F}$ & $T$ \\
$F$ & $\mathbf{T}$ & $\mathbf{T}$ & $\mathbf{F}$ & $\mathbf{T}$ & $T$ \\
$D P$ & $\mathbf{T}$ & $\mathbf{T}$ & $T$ & $T$ & $T$ \\
$G F$ & $\mathbf{F}$ & $F$ & $\mathbf{T}$ & $\mathbf{T}$ & $T$ \\
\hline
\end{tabular}

Looking at Table 1, one can observe that the two ranking methods based on iterative application of a choice function, namely $I t . N F R$ and $I t . M i F$, lack monotonicity property. This observation is concordant with the work of Bouyssou [19]. Moreover, all ranking methods have property $C C$, which guaranties that when they are applied to exploitation of fuzzy relation $\mathcal{R}(29)$, they produce final rankings respecting dominance relation $D$ over set $A$.

Further analysis of the properties presented in Table 1 leads to the conclusion that, in view of the considered list of desirable properties, the best ranking method for exploitation of a fuzzy relation is the $N F R$ method. This is because it satisfies most of the properties (which is, however, true also for the $L / E$ ranking method) and, moreover, satisfies the first five properties (i.e., $N, M, C C, I N D O$, and $I C$ ). The lack of property $O$ is alleviated by the fact that values $\mathcal{R}(a, b), a, b \in A$, may be interpreted in "cardinal" terms. This is due to the definition of relation $\mathcal{R}(29)$, the way of constructing relations $\mathcal{S}$ and $\mathcal{S}^{c}$, and the semantics of values $\widehat{\epsilon}_{T}\left(r_{T}\right)$ (i.e., relative number of "negative pairs of objects" covered by rule $\left.r_{T}\right)$.

It is worth pointing out that the $N F R$ ranking method is attractive not only because of the desirable properties it possesses. It represents an intuitive way of reasoning about relative worth of objects in set $A$, as it takes into account both positive and negative arguments concerning each object (i.e., strength and weakness of each object), as advocated by Fortemps and Słowiński [36].

\section{Illustrative Example}

Let us consider the data set analyzed by Andenmatten [1], concerning performance of chosen international airlines, in terms of the following financial ratios: 
(1) Benefit Margin (BM),

(2) Interest Coverage (IC),

(3) Debt Payback (DP),

(4) Shareholder Funds/Fixed Assets (SHF/FA),

(5) Equity Ratio (ER).

The precise meaning of the above ratios can be found in [1]. Values of the considered financial ratios were gathered for minimum five and maximum ten consecutive years. Then, for each airline and each ratio, an average for each five successive years was calculated. In result, one got a set of 49 airline-year objects, denoted by $a_{1}, \ldots, a_{49}$. Each object was described by the following 10 criteria:

$\left(g_{1}\right)$ Average Benefit Margin (Avg.BM),

$\left(g_{2}\right)$ Average Interest Coverage (Avg.IC),

$\left(g_{3}\right)$ Average Debt Payback (Avg.DP),

$\left(g_{4}\right)$ Average Shareholder Funds/Fixed Assets (Avg.SHF/FA),

$\left(g_{5}\right)$ Average Equity ratio (Avg.ER),

$\left(g_{6}\right)$ Benefit Margin (BM),

$\left(g_{7}\right)$ Interest Coverage (IC),

$\left(g_{8}\right)$ Debt Payback (DP),

$\left(g_{9}\right)$ Shareholder Funds/Fixed Assets (SHF/FA),

$\left(g_{10}\right)$ Equity Ratio (ER).

All criteria, apart from Avg.DP and DP, are of gain-type, i.e., the higher the criterion value the better. Criteria Avg.DP and DP are cost-type criteria, i.e., the smaller the criterion value the better. Given an airline-year object, e.g., delta-89, values of the first five criteria are averages for the period 1985-1989, while values of the last five criteria are values of the respective financial ratios from the year 1989.

In [1], each airline was assigned to one of five ordered categories (reflecting investment risk), depending on its rating scores according to Moody's and Standard\&Poor's. The categories were defined as shown in Table 2. The order of the categories was: $U I G \succ L I G \succ U N I G \succ L N I G \succ S p G$. Thus, category $U I G$

Table 2: Definition of the considered categories of airlines

\begin{tabular}{ccc}
\hline Category & Moody's score & Standard\&Poor's score \\
\hline$U I G$ & $\mathrm{Aaa}, \mathrm{Aa}$ & $\mathrm{AAA}, \mathrm{AA}$ \\
$L I G$ & $\mathrm{~A}, \mathrm{Baa}$ & $\mathrm{A}, \mathrm{BBB}$ \\
$U N I G$ & $\mathrm{Ba}, \mathrm{B}$ & $\mathrm{BB}, \mathrm{B}$ \\
$L N I G$ & $\mathrm{Caa}, \mathrm{Ca}$ & $\mathrm{CCC}, \mathrm{CC}$ \\
$S p G$ & $\mathrm{C}$ & $\mathrm{C}, \mathrm{D}$ \\
\hline
\end{tabular}

is the best one (it reflects the lowest investment risk), and category $S p G$ is the worst one (it reflects the highest investment risk).

The evaluations of all 49 airline-year objects on the 10 considered criteria, as well as assignments of these objects to the 5 considered categories, are given in the Appendix (Table 10). Just like in [1], the objects are ordered by categories. This should not be a concern since the order of objects does not influence the results 
obtained using the proposed approach to multicriteria raking. Let us observe that for the set of criteria $G=\left\{g_{1}, \ldots, g_{10}\right\}$ it is not true that the better the evaluation of an object on considered criteria, the more this object is preferable to another object. For instance, object $a_{17}$ assigned to category $L I G$ dominates object $a_{4}$ which is assigned to better category UIG. This may be caused by the lack of some important criteria. In total, there are 16 out of 49 objects for which given assignment to a category is inconsistent with the dominance relation $D$ over the set of all 49 objects. Let us denote by $A_{\text {inc }}$ the set composed of these 16 objects. Then, we have $A_{\text {inc }}=\left\{a_{4}, a_{9}, a_{10}, a_{17}, a_{25}, a_{26}, a_{30}, a_{31}, a_{32}, a_{34}, a_{35}, a_{37}, a_{38}, a_{40}, a_{42}, a_{45}\right\}$.

We consider that all the 49 airline-year objects constitute set $A$ of objects to be ranked. Moreover, for the purpose of the example, we choose 11 airline-year objects from the set $A \backslash A_{\text {inc }}$ to be reference objects, constituting set $A^{R} \subseteq A$. We select these 11 objects in such a way, that all categories but $L I G$ have two "representatives", i.e., for each category $C l \in\{U I G, U N I G, L N I G, S p G\}$, there exist exactly two airlineyear objects $a, b \in A^{R}$, such that the airlines of objects $a$ and $a$ belong to category $C l$. From the most numerous category $L I G$, we choose three "representatives". The reference objects are shown in Table 3 . Let

\begin{tabular}{|c|c|c|}
\hline Id & Airline-year & Category \\
\hline$a_{1}$ & japan-89 & $U I G$ \\
\hline$a_{2}$ & japan-90 & $U I G$ \\
\hline$a_{16}$ & japan-93 & $L I G$ \\
\hline$a_{21}$ & stw-91 & $L I G$ \\
\hline$a_{27}$ & usair-88 & $L I G$ \\
\hline$a_{33}$ & alaska-91 & $U N I G$ \\
\hline$a_{36}$ & usair-92 & $U N I G$ \\
\hline$a_{41}$ & panam-88 & $L N I G$ \\
\hline$a_{43}$ & united-88 & $L N I G$ \\
\hline$a_{44}$ & metro-89 & $S p G$ \\
\hline$a_{48}$ & metro-91 & $S p G$ \\
\hline
\end{tabular}

us observe that the assignment of airlines to considered categories can be used as a source of preference information. Therefore, given any two airline-year objects $a, b \in A^{R}$, we fix $a S b$ whenever the category of the airline of object $a$ is not worse than the category of the airline of object $b$. Moreover, we fix $a S^{c} b$ whenever the category of the airline of object $a$ is strictly worse than the category of the airline of object $b$. In this way, $B=A^{R} \times A^{R}$.

Given the preference information, the following calculations are performed using jRank ${ }^{1}$ software [88]. For the sake of simplicity, we make an assumption that all the considered criteria are cardinal ones.

The preference information in the form of pairwise comparisons of 11 reference objects yields a PCT composed of 121 pairs of objects. A part of this PCT is shown in Table 4. Let us note that the cardinality of relation $S$ is 73 , and the cardinality of relation $S^{c}$ is 48 .

One can observe in the PCT several inconsistencies w.r.t. dominance relation $D_{2}$ over set $B$. Such inconsistency occurs when a pair of objects $(a, b) \in S$ is dominated by a pair of objects $(c, d) \in S^{c}$. Inconsistent pairs of objects appearing in Table 4 are marked in this table by symbol *. All inconsistencies w.r.t. dominance relation $D_{2}$ over $B$ are also presented in Table 5 , where symbol $*$ indicates where pair $(a, b) \in S$ from the corresponding row is inconsistent with pair $(c, d) \in S^{c}$ from the corresponding column.

In order to show potential advantage of VC-DRSA over DRSA, when applied to preference learning in multicriteria raking, we consider two independent calculation paths, taking the PCT shown in Table 4 as a "point of departure". Both paths are composed of the following steps:

$\left(s_{1}\right)$ calculation of lower approximations of relations $S$ and $S^{c}$, according to definitions (14) and (15), respectively,

${ }^{1}$ See http://www.cs.put.poznan.pl/mszelag/Software/jRank/jRank.html. 
Table 4: A part of the PCT yielded by pairwise comparisons of 11 airline-year reference objects

\begin{tabular}{|c|c|c|c|c|c|c|c|c|c|c|c|}
\hline$(a, b)$ & $\Delta_{1}$ & $\Delta_{2}$ & $\Delta_{3}$ & $\Delta_{4}$ & $\Delta_{5}$ & $\Delta_{6}$ & $\Delta_{7}$ & $\Delta_{8}$ & $\Delta_{9}$ & $\Delta_{10}$ & Relation \\
\hline$\left(a_{1}, a_{1}\right)$ & 0 & 0 & 0 & 0 & 0 & 0 & 0 & 0 & 0 & 0 & $S$ \\
\hline$\left(a_{1}, a_{2}\right)$ & 0.08 & -0.03 & -1.6 & -7.23 & -1.6 & 34.53 & 9.18 & -14.0 & -22.2 & -3.0 & $S$ \\
\hline$\ldots$ & $\ldots$ & $\ldots$ & $\ldots$ & $\ldots$ & $\ldots$ & $\ldots$ & $\ldots$ & $\ldots$ & $\ldots$ & $\ldots$ & $\ldots$ \\
\hline$\left(a_{1}, a_{48}\right)$ & 17.1 & 4.78 & -15.3 & 50.15 & 21.0 & 56.41 & 17.0 & 1.1 & 235.0 & 104.8 & $S$ \\
\hline$\left(a_{2}, a_{1}\right)^{*}$ & -0.08 & 0.03 & 1.6 & 7.23 & 1.6 & -34.53 & -9.18 & 14.0 & 22.2 & 3.0 & $S$ \\
\hline$\left(a_{2}, a_{2}\right)$ & 0 & 0 & 0 & 0 & 0 & 0 & 0 & 0 & 0 & 0 & $S$ \\
\hline$\left(a_{2}, a_{16}\right)$ & 3.1 & 0.78 & -13.7 & -12.21 & -2.9 & 6.33 & 2.18 & -4.7 & 32.5 & 9.2 & $S$ \\
\hline$\left(a_{2}, a_{21}\right)^{*}$ & -0.55 & 0.8 & 3.5 & -12.37 & -23.5 & -5.78 & -0.62 & 10.5 & 25.8 & -9.1 & $S$ \\
\hline$\left(a_{2}, a_{27}\right)^{*}$ & 1.6 & 0.33 & 3.6 & -25.47 & -25.2 & -5.2 & -0.93 & 12.6 & 9.9 & -13.6 & $S$ \\
\hline$\ldots$ & . & $\ldots$ & $\ldots$ & $\ldots$ & $\ldots$ & $\ldots$ & . & $\ldots$ & $\ldots$ & $\ldots$ & $\ldots$ \\
\hline$\left(a_{16}, a_{1}\right)$ & -3.18 & -0.75 & 15.3 & 19.44 & 4.5 & -40.86 & -11.36 & 18.7 & -10.3 & -6.2 & $S^{c}$ \\
\hline$\left(a_{16}, a_{2}\right)$ & -3.1 & -0.78 & 13.7 & 12.21 & 2.9 & -6.33 & -2.18 & 4.7 & -32.5 & -9.2 & $S^{c}$ \\
\hline$\left(a_{16}, a_{16}\right)$ & 0 & 0 & 0 & 0 & 0 & 0 & 0 & 0 & 0 & 0 & $S$ \\
\hline$\left(a_{16}, a_{21}\right)^{*}$ & -3.65 & 0.02 & 17.2 & -0.16 & -20.6 & -12.11 & -2.8 & 15.2 & -6.7 & -18.3 & $S$ \\
\hline$\left(a_{16}, a_{27}\right)^{*}$ & -1.5 & -0.45 & 17.3 & -13.26 & -22.3 & -11.53 & -3.11 & 17.3 & -22.6 & -22.8 & $S$ \\
\hline$\left(a_{16}, a_{33}\right)^{*}$ & 2.58 & 0.44 & 15.9 & -3.78 & -13.1 & -8.86 & -2.12 & 12.8 & 0.7 & -7.6 & $S$ \\
\hline$\ldots$ & $\cdots$ & $\ldots$ & $\cdots$ & $\cdots$ & $\cdots$ & $\ldots$ & $\ldots$ & $\cdots$ & $\ldots$ & $\cdots$ & $\cdots$ \\
\hline$\left(a_{21}, a_{2}\right)^{*}$ & 0.55 & -0.8 & -3.5 & 12.37 & 23.5 & 5.78 & 0.62 & -10.5 & -25.8 & 9.1 & $S^{c}$ \\
\hline$\ldots$ & $\ldots$ & $\ldots$ & $\cdots$ & $\cdots$ & $\cdots$ & $\ldots$ & $\ldots$ & $\cdots$ & $\ldots$ & $\ldots$ & $\ldots$ \\
\hline$\left(a_{27}, a_{2}\right)^{*}$ & -1.6 & -0.33 & -3.6 & 25.47 & 25.2 & 5.2 & 0.93 & -12.6 & -9.9 & 13.6 & $S^{c}$ \\
\hline$\ldots$ & $\ldots$ & $\ldots$ & $\ldots$ & $\ldots$ & $\ldots$ & $\ldots$ & $\ldots$ & $\ldots$ & $\ldots$ & $\ldots$ & $\ldots$ \\
\hline$\left(a_{33}, a_{16}\right)^{*}$ & -2.58 & -0.44 & -15.9 & 3.78 & 13.1 & 8.86 & 2.12 & -12.8 & -0.7 & 7.6 & $S^{c}$ \\
\hline$\ldots$ & $\ldots$ & $\ldots$ & $\ldots$ & $\ldots$ & $\ldots$ & $\ldots$ & $\ldots$ & $\ldots$ & $\ldots$ & $\ldots$ & $\cdots$ \\
\hline$\left(a_{36}, a_{33}\right)^{*}$ & -3.17 & -1.57 & 15.9 & -23.18 & -13.2 & 2.66 & 0.74 & -3.0 & -38.7 & -26.1 & $S$ \\
\hline$\ldots$ & $\ldots$ & $\ldots$ & $\ldots$ & $\ldots$ & $\ldots$ & $\ldots$ & $\ldots$ & $\ldots$ & $\ldots$ & $\ldots$ & $\ldots$ \\
\hline$\left(a_{36}, a_{43}\right)^{*}$ & -2.68 & -1.49 & 13.5 & -16.14 & -5.1 & 1.26 & -1.1 & 0.6 & -35.1 & -20.9 & $S$ \\
\hline$\ldots$ & . & $\ldots$ & $\ldots$ & $\ldots$ & $\ldots$ & $\ldots$ & $\ldots$ & $\ldots$ & $\ldots$ & $\ldots$ & $\ldots$ \\
\hline$\left(a_{41}, a_{43}\right)^{*}$ & -6.12 & -2.37 & -6.0 & -44.95 & -25.9 & -1.46 & -1.45 & 0.7 & -58.3 & -32.5 & $S$ \\
\hline$\cdots$ & $\begin{array}{l}\ldots \\
307\end{array}$ & $\begin{array}{l}\cdots \\
05\end{array}$ & $\begin{array}{l}\cdots \\
125\end{array}$ & $\begin{array}{l}\ldots \\
326\end{array}$ & $\cdots$ & $\begin{array}{c}\cdots \\
1026\end{array}$ & & $\cdots$ & $\cdots$ & $\cdots$ & $\cdots$ \\
\hline$\left(a_{43}, a_{16}\right)^{*}$ & -3.07 & -0.52 & -13.5 & -3.26 & 5.0 & 10.26 & 3.96 & -16.4 & -4.3 & 2.4 & $S^{c}$ \\
\hline$\cdots$ & $\cdots$ & ... & $\ldots$ & $\ldots$ & $\cdots$ & $\cdots$ & $\cdots$ & $\cdots$ & $\cdots$ & $\cdots$ & $\ldots$ \\
\hline & -0.49 & -0.08 & 2.4 & -7.04 & -8.1 & 1.4 & 1.84 & -3.6 & -3.6 & -5.2 & $S^{c}$ \\
\hline$\left(a_{43}, a_{36}\right)^{*}$ & 2.68 & 1.49 & -13.5 & 16.14 & 5.1 & -1.26 & 1.1 & -0.6 & 35.1 & 20.9 & $S^{c}$ \\
\hline$\ldots$ & $\ldots$ & $\ldots$ & $\ldots$ & $\ldots$ & $\ldots$ & $\ldots$ & $\ldots$ & $\cdots$ & $\ldots$ & $\ldots$ & $\ldots$ \\
\hline$\left(a_{48}, a_{1}\right)$ & -17.1 & -4.78 & 15.3 & -50.15 & -21.0 & -56.41 & -17.0 & -1.1 & -235.0 & -104.8 & $S^{c}$ \\
\hline$\ldots$ & $\ldots$ & $\ldots$ & $\ldots$ & $\ldots$ & $\ldots$ & $\ldots$ & $\ldots$ & $\ldots$ & $\ldots$ & $\ldots$ & $\cdots$ \\
\hline$\left(a_{48}, a_{43}\right)$ & -10.85 & -3.51 & 13.5 & -66.33 & -30.5 & -25.81 & -9.6 & -3.4 & -220.4 & -101.0 & $S^{c}$ \\
\hline$\left(a_{48}, a_{44}\right)$ & -8.79 & -3.81 & 17.9 & -61.28 & -32.1 & -8.72 & -3.63 & -19.8 & -214.2 & -96.4 & $S$ \\
\hline$\left(a_{48}, a_{48}\right)$ & 0 & 0 & 0 & 0 & 0 & 0 & 0 & 0 & 0 & 0 & $S$ \\
\hline
\end{tabular}

$\left(s_{2}\right)$ calculation of a minimal set of decision rules by VC-DomLEM algorithm,

$\left(s_{3}\right)$ application of the induced rules on set $A$,

$\left(s_{4}\right)$ exploitation of the resulting preference structure using the $N F R$ ranking method,

$\left(s_{5}\right)$ evaluation of the obtained final ranking over set $A$. 
Table 5: Inconsistencies in the PCT yielded by pairwise comparisons of reference objects

\begin{tabular}{|c|c|c|c|c|c|c|}
\hline()$^{*} \in S \downarrow \|()^{*} \in S^{c} \rightarrow$ & $\left(a_{21}, a_{2}\right)$ & $\left(a_{27}, a_{2}\right)$ & $\left(a_{33}, a_{16}\right)$ & $\left(a_{43}, a_{16}\right)$ & $\left(a_{43}, a_{33}\right)$ & $\left(a_{43}, a_{36}\right)$ \\
\hline$\left(a_{2}, a_{1}\right)$ & & & & & & * \\
\hline$\left(a_{2}, a_{21}\right)$ & & & & & & * \\
\hline$\left(a_{2}, a_{27}\right)$ & & & & & & * \\
\hline$\left(a_{16}, a_{21}\right)$ & & & & & & * \\
\hline$\left(a_{16}, a_{27}\right)$ & & & & & $*$ & * \\
\hline$\left(a_{16}, a_{33}\right)$ & & & & & & $*$ \\
\hline$\left(a_{36}, a_{33}\right)$ & & * & $*$ & * & & \\
\hline$\left(a_{36}, a_{43}\right)$ & * & * & * & & & \\
\hline$\left(a_{41}, a_{43}\right)$ & & & $*$ & $*$ & & $*$ \\
\hline
\end{tabular}

Each of the above steps "produces" some results. These are: lower approximations of outranking and non-outranking relations obtained in step $\left(s_{1}\right)$, sets $R_{S}$ and $R_{S^{c}}$ of minimal decision rules obtained in step $\left(s_{2}\right)$, preference structure on $A$ obtained in step $\left(s_{3}\right)$, final ranking (weak order) on $A$ obtained in step $\left(s_{4}\right)$, and value of a chosen error measure obtained in step $\left(s_{5}\right)$. However, these results differ in both calculation paths only due to decisions made in step $\left(s_{1}\right)$, concerning consistency thresholds $\theta_{S}$ and $\theta_{S^{c}}$ used to define lower approximations (14) and (15). In the first path, denoted by $c p_{\theta=0}$, we assume that both consistency thresholds are equal to zero. Thus, calculated lower approximations are the same as in the case of DRSA $[44,46,85]$. In the second path, denoted by $c p_{\theta>0}$, we choose $\theta_{S}=\theta_{S^{c}}=0.042$. In this way, we relax a little bit the conditions for inclusion of pairs of objects to lower approximations (14) and (15). In particular, a pair of objects $(a, b) \in S$ is considered to be sufficiently consistent (and thus included in $\underline{S}$ ) if it is dominated by at most two pairs of objects belonging to relation $S^{c}$ (this can be verified using Equation (12): $\left.2 / 48=0.0417<\theta_{S}=0.042<3 / 48=0.0625\right)$. Moreover, a pair of objects $(a, b) \in S^{c}$ is considered to be sufficiently consistent (and thus included in $\underline{S^{c}}$ ) if it dominates at most three pairs of objects belonging to relation $S$ (this can be verified using Equation (13): $3 / 73=0.0411<\theta_{S^{c}}=0.042<4 / 73=0.0548$ ).

In step $\left(s_{5}\right)$, we employ dependencies $(4),(5)$, and (6), to represent pairwise comparisons of objects in terms of relations $P, P^{-1}$, and $I$. Moreover, we use the following representation of a final ranking $\succeq(A, \mathcal{R})$ in terms of relations $P_{\succeq}, P_{\succeq}^{-1}$, and $I_{\succeq}$ :

$$
\begin{aligned}
a P_{\succeq} b & \Leftrightarrow a \succeq(A, \mathcal{R}) b \text { and not } b \succeq(A, \mathcal{R}) a, \\
a P_{\succeq}^{-1} b & \Leftrightarrow \text { not } a \succeq(A, \mathcal{R}) b \text { and } b \succeq(A, \mathcal{R}) a, \\
a I_{\succeq} b & \Leftrightarrow a \succeq(A, \mathcal{R}) b \text { and } b \succeq(A, \mathcal{R}) a,
\end{aligned}
$$

where $a, b \in A$. Thus, $a P_{\succeq} b$ iff object $a$ is ranked higher than object $b, a P_{\succeq}^{-1} b$ iff object $a$ is ranked lower than object $b$, and $a I_{\succ} b$ iff the ranks of objects $a$ and $b$ are equal. Then, we apply a modified Kendall rank correlation coefficient $\tau^{\prime}$ to measure:

- the concordance between a final ranking and the pairwise comparisons of reference objects, derived from Table 3,

- the concordance between a final ranking and the pairwise comparisons of all objects from set $A$, derived from Table 10.

The modifications of the Kendall rank correlation coefficient are twofold. First, we do not count an error when a pair of objects is not reassigned to indifference relation. More precisely, if $a I b$, we do not expect that $a I_{\succ} b$, for any $a, b \in A$. Thus, there is no error if objects from a given category do not belong to the same rank, but instead are ranked one after another, without "interference" of objects from other categories. Second, when calculating an error, we do not take into account pairs of objects which cannot be reassigned to preference relation or inverse preference relation because their original assignment was inconsistent with dominance relation $D$ over set $A$. More precisely: 
- if $a P b$ but $b D a$, then we do not expect that $a P_{\succeq} b$; in fact, since the $N F R$ ranking method has property $C C$, due to Corollary 1 , we will obtain $a P_{\succeq}^{-1} b$ or $a I_{\succeq} b$,

- if $a P^{-1} b$ but $a D b$, then we do not expect that $a P_{\succeq}^{-1} b$; in fact, we will obtain $a P_{\succeq} b$ or $a I_{\succeq} b$,

where $a, b \in A$. Thus, by the second modification, we admit that the discrepancies between relations $P$ and $P_{\succeq}$, as well as between relations $P^{-1}$ and $P_{\succ}^{-1}$, resulting from the respect of dominance relation $D$ over set $A$, indicate the "weakness" of the preference information or the "weakness" of the considered set of criteria (e.g., lack of some important criteria) rather than the "weakness" of the final ranking.

Let use denote by $P_{E}$ the set of pairs of objects that are expected to be preserved in the preference relation. This set is defined as:

$$
\left.P_{E}=P \backslash\{(a, b) \in A \times A: b D a)\right\} .
$$

Then, the modified Kendall rank correlation coefficient $\tau^{\prime}$, applied to evaluate ranking $\succeq(A, \mathcal{R})$ (represented in terms of relations $P_{\succeq}, P_{\succeq}^{-1}$, and $I_{\succeq}$ ) w.r.t. given pairwise comparisons (represented in terms of relations $P, P^{-1}$, and $I$ ), is described by the following equation:

$$
\tau^{\prime}\left(P_{\succeq}, P_{\succeq}^{-1}, I_{\succeq}, P_{E}\right)=1-2 \frac{\sum_{(a, b) \in P_{E}} \operatorname{err}(a, b)}{\left|P_{E}\right|},
$$

where $\operatorname{err}(a, b)$ denotes an error accounted for a pair of objects $(a, b) \in P_{E}$, defined as:

$$
\operatorname{err}(a, b)= \begin{cases}0, & \text { if } a P_{E} b \text { and } a P_{\succeq} b \\ \frac{1}{2}, & \text { if } a P_{E} b \text { and } a I_{\succeq}^{b} \\ 1, & \text { if } a P_{E} b \text { and } a P_{\succeq}^{-1} b\end{cases}
$$

It is easy to see that coefficient $\tau^{\prime}$ belongs to the interval $[-1,1]$. The best possible value of $\tau^{\prime}$ is 1 , and the worst possible value is -1 .

We distinguish two values of $\tau^{\prime}$ :

- $\tau_{r e f}^{\prime}$, quantifying concordance between a final ranking and the pairwise comparisons of reference objects,

- $\tau_{\text {all }}^{\prime}$, quantifying concordance between a final ranking and the pairwise comparisons of all objects from set $A$.

Value $\tau_{r e f}^{\prime}$ is obtained from Equation (50) for relations $P$ and $P^{-1}$ representing pairwise comparisons of reference objects. Value $\tau_{\text {all }}^{\prime}$ is obtained from Equation (50) for relations $P$ and $P^{-1}$ representing pairwise comparisons of all objects from set $A$.

Table 6 summarizes the results obtained in subsequent steps $\left(s_{1}\right)-\left(s_{5}\right)$, along both calculations paths.

Table 6: Summary of results obtained in steps $\left(s_{1}\right)-\left(s_{5}\right)$, for calculations paths $c p_{\theta}=0, c p_{\theta>0}$

\begin{tabular}{ccccccc}
\hline Calculation path & $|\underline{S}|$ & $\left|\underline{S^{c}}\right|$ & $\left|R_{S}\right|$ & $\left|R_{S^{c}}\right|$ & $\tau_{\text {ref }}^{\prime}$ & $\tau_{\text {all }}^{\prime}$ \\
\hline$c p_{\theta=0}$ & 64 & 42 & 7 & 10 & 0.792 & 0.650 \\
$c p_{\theta>0}$ & 70 & 47 & 5 & 7 & 0.875 & 0.812 \\
\hline
\end{tabular}

Looking at Table 6 , it is clear that the results obtained along calculation path $c p_{\theta>0}$ are better (greater lower approximations, smaller amounts of rules, and higher values of $\tau^{\prime}$ ). Thus, for the considered illustrative example, proposed VC-DRSA proved to be more useful than DRSA, when applied to preference learning in multicriteria ranking. In view of this conclusion, in the following, we present only results obtained along calculation path $c p_{\theta>0}$. 
Table 7: Minimal decision rules induced by VC-DomLEM algorithm

\begin{tabular}{|c|c|c|c|}
\hline Decision rule $r_{T}$ & $\widehat{\epsilon}_{T}\left(r_{T}\right)$ & $c f\left(r_{T}\right)$ & $\sigma\left(r_{T}\right)$ \\
\hline if $\left(\Delta_{6}(a, b) \geq 11.53\right)$, then $a S b$ & 0 & $27 / 73=0.370$ & 0.370 \\
\hline if $\left(\Delta_{1}(a, b) \geq-8.79\right) \wedge\left(\Delta_{8}(a, b) \leq-19.8\right)$, then $a S b$ & 0 & $1 / 73=0.014$ & 0.014 \\
\hline if $\left(\Delta_{8}(a, b) \leq 14.0\right) \wedge\left(\Delta_{9}(a, b) \geq 22.2\right)$, then $a S b$ & 0.021 & $29 / 73=0.397$ & 0.389 \\
\hline if $\left(\Delta_{2}(a, b) \geq 0.02\right) \wedge\left(\Delta_{6}(a, b) \geq-12.11\right)$, then $a S b$ & 0.021 & $49 / 73=0.671$ & 0.657 \\
\hline if $\left(\Delta_{1}(a, b) \geq-1.5\right) \wedge\left(\Delta_{6}(a, b) \geq-11.53\right) \wedge\left(\Delta_{9}(a, b) \geq-22.6\right)$, then $a S b$ & 0.042 & $64 / 73=0.877$ & 0.840 \\
\hline if $\left(\Delta_{7}(a, b) \leq-4.13\right) \wedge\left(\Delta_{9}(a, b) \leq 12.3\right)$, then $a S^{c} b$ & 0 & $22 / 48=0.458$ & 0.458 \\
\hline if $\left(\Delta_{3}(a, b) \geq 1.6\right) \wedge\left(\Delta_{6}(a, b) \leq-15.63\right)$, then $a S^{c} b$ & 0 & $11 / 48=0.229$ & 0.229 \\
\hline if $\left(\Delta_{7}(a, b) \leq-4.87\right) \wedge\left(\Delta_{8}(a, b) \geq 15.8\right)$, then $a S^{c} b$ & 0 & $5 / 48=0.104$ & 0.104 \\
\hline if $\left(\Delta_{2}(a, b) \leq-0.52\right) \wedge\left(\Delta_{7}(a, b) \leq 3.96\right) \wedge\left(\Delta_{8}(a, b) \geq-16.4\right)$, then $a S^{c} b$ & 0.041 & $34 / 48=0.708$ & 0.679 \\
\hline if $\left(\Delta_{1}(a, b) \leq-2.58\right) \wedge\left(\Delta_{2}(a, b) \leq-0.44\right) \wedge\left(\Delta_{8}(a, b) \geq-12.8\right)$, then $a S^{c} b$ & 0.041 & $30 / 48=0.625$ & 0.599 \\
\hline if $\left(\Delta_{1}(a, b) \leq-1.6\right) \wedge\left(\Delta_{2}(a, b) \leq-0.33\right) \wedge\left(\Delta_{8}(a, b) \geq-12.6\right)$, then $a S^{c} b$ & 0.041 & $31 / 48=0.646$ & 0.619 \\
\hline$\left(\Delta_{8}(a, b) \geq-3.6\right)$, then $a S^{c} b$ & 0.041 & $26 / 48=0.542$ & 0.519 \\
\hline
\end{tabular}

The set of minimal decision rules induced by VC-DomLEM algorithm is presented in Table 7, where each rule $r_{T}$ is characterized in terms of three statistics: rule consistency measure $\widehat{\epsilon}_{T}\left(r_{T}\right)$, coverage factor $c f\left(r_{T}\right)$ (i.e., the ratio of the number of pairs of objects supporting $r_{T}$ and the cardinality of relation $T$ ), and strength $\sigma\left(r_{T}\right)$ calculated according to $(21)$. It is worth noting that the rules are relatively short and the amount of rules is small w.r.t. the size of the PCT. Moreover, the rules are easy to understand. For example, the first rule from Table 7 is read as follows: if the difference of Benefit Margin for airline-year $a$ and airline-year $b$ is at least 11.53 , then $a$ is weakly preferred to $b$.

Final ranking of all objects from set $A$, obtained using the $N F R$ ranking method, is presented in Table 8, where each object is located in a cell corresponding to its ranking position (rank) and category, and reference objects are marked in bold; moreover, for each rank we give respective net flow score which is the value of scoring function $S^{N F}(28)$. Now, let us analyze how we obtained $\tau_{\text {ref }}^{\prime}=0.875$. First, due to the fact that $A^{R} \subseteq A \backslash A_{\text {inc }}$, from (49) we get $P_{E}=P$. Second, according to (51), we account three times error value 1, since $a_{36} P a_{43}$ but $a_{36} P_{\succeq}^{-1} a_{43}, a_{36} P a_{44}$ but $a_{36} P_{\succeq}^{-1} a_{44}$, and $a_{41} P a_{44}$ but $a_{41} P_{\succeq}^{-1} a_{44}$. Third, $|P|=48$. Thus, from (50) we get $\tau_{\text {ref }}^{\prime}=1-2 \frac{3}{48}=0.875$. Since this value is close to 1 , we can say that the induced set of decision rules is a good preference model.

\section{Summary and Conclusions}

We presented a methodology for non-statistical preference learning in multicriteria ranking. In this methodology, the preference information, given by a decision maker (DM) in the form of pairwise comparisons of some reference objects, is used to learn a preference model in the form of a set of "if ..., then ..." decision rules.

We considered that for any two different reference objects $a, b$, the DM can declare either "object $a$ is at least as good as object $b$ " or "object $a$ is not at least as good as object $b$ ", or abstain from any judgment. In this way, the DM specifies two comprehensive preference relations. Such preference information is used to create a pairwise comparison table (PCT). As the pairwise comparisons given by the DM are prone to inconsistencies, we proposed to structure them using Variable Consistency Dominance-based Rough Set Approach (VC-DRSA), by calculation of lower approximations of the two comprehensive preference relations. In this way, we can restrict a priori the set of pairs of objects used during induction of decision rules to a subset of sufficiently consistent pairs of objects. This restriction is motivated by the goal of learning a "reliable" preference model.

Application of induced decision rules on the whole set of objects to be ranked yields a fuzzy preference structure (directed weighted graph) on this set. This structure is then exploited using a ranking method, in 
Table 8: Final ranking of all objects from set $A$, obtained using the $N F R$ ranking method

\begin{tabular}{|c|c|c|c|c|c|c|}
\hline Rank & Net flow score & $U I G$ & $L I G$ & $U N I G$ & $L N I G$ & $S p G$ \\
\hline 1 & 66.404 & $a_{1}$ & & & & \\
\hline 2 & 63.981 & & $a_{17}$ & & & \\
\hline 3 & 54.352 & & $a_{11}$ & & & \\
\hline 4 & 48.267 & $\mathbf{a}_{2}$ & & & & \\
\hline 5 & 44.741 & $a_{3}$ & & & & \\
\hline 6 & 44.088 & & $a_{12}$ & & & \\
\hline 7 & 42.983 & & $a_{5}$ & & & \\
\hline 8 & 40.847 & $a_{4}$ & & & & \\
\hline 9 & 40.091 & & $a_{20}$ & & & \\
\hline 10 & 39.808 & & $a_{19}$ & & & \\
\hline 11 & 39.497 & & $a_{22}$ & & & \\
\hline 12 & 39.353 & & $a_{18}$ & & & \\
\hline 13 & 36.643 & & $a_{13}$ & & & \\
\hline 14 & 35.281 & & $a_{6}$ & & & \\
\hline 15 & 34.604 & & $a_{27}$ & & & \\
\hline 16 & 29.84 & & $\mathbf{a}_{21}$ & & & \\
\hline 17 & 25.285 & & & & $a_{40}$ & \\
\hline 18 & 22.029 & & $a_{14}$ & & & \\
\hline 19 & 17.208 & & $a_{15}$ & & & \\
\hline 20 & 13.564 & & $a_{7}$ & & & \\
\hline 21 & 10.781 & & & $a_{30}$ & & \\
\hline 22 & 6.08 & & & $a_{32}$ & & \\
\hline 23 & 2.977 & & $a_{8}$ & & & \\
\hline 24 & 2.106 & & & $a_{31}$ & & \\
\hline 25 & 1.152 & & $a_{24}$ & & & \\
\hline 26 & 0.168 & & $a_{28}$ & & & \\
\hline 27 & 0.109 & & $a_{16}$ & & & \\
\hline 28 & -5.856 & & $a_{10}$ & & & \\
\hline 29 & -8.791 & & $a_{25}$ & & & \\
\hline 30 & -9.47 & & & & $a_{37}$ & \\
\hline 31 & -12.009 & & $a_{9}$ & & & \\
\hline 32 & -14.919 & & $a_{23}$ & & & \\
\hline 33 & -15.523 & & & $a_{33}$ & & \\
\hline 34 & -18.336 & & & & $a_{38}$ & \\
\hline 35 & -21.323 & & & & $a_{43}$ & \\
\hline 36 & -28.948 & & $a_{26}$ & & & \\
\hline 37 & -29.483 & & $a_{29}$ & & & \\
\hline 38 & -32.125 & & & & $a_{39}$ & \\
\hline 39 & -32.376 & & & $a_{34}$ & & \\
\hline 40 & -42.326 & & & & & $a_{44}$ \\
\hline 41 & -46.797 & & & $a_{35}$ & & \\
\hline 42 & -49.338 & & & $a_{36}$ & & \\
\hline 43 & -52.647 & & & & & $a_{45}$ \\
\hline 44 & -55.619 & & & & $\mathbf{a}_{41}$ & \\
\hline 45 & -62.441 & & & & & $a_{47}$ \\
\hline 46 & -62.468 & & & & & $a_{46}$ \\
\hline 47 & -64.237 & & & & & $a_{49}$ \\
\hline 48 & -67.453 & & & & $a_{42}$ & \\
\hline 49 & -69.753 & & & & & $a_{48}$ \\
\hline
\end{tabular}


order to work out a final recommendation, i.e., a ranking of objects. We proposed a list of some desirable properties that a ranking method is expected to have, and compared several ranking methods studied in the literature w.r.t. these properties. Based on this comparison, we chose one ranking method, the Net Flow Rule (NFR), enjoying the most desirable properties.

We illustrated the proposed methodology by an example concerning performance of chosen international airlines. This example shows usefulness of the proposed methodology for dealing with multicriteria ranking problems.

\section{Acknowledgements}

The first author wishes to acknowledge financial support from the Poznań University of Technology, grant no. 91-516/DS-MLODA KADRA. Moreover, the first author declares that he is a scholarship holder within the 2012/2013 project "Scholarship support for PH.D. students specializing in majors strategic for Wielkopolska's development", Sub-measure 8.2.2 of Human Capital Operational Programme, co-financed by European Union under the European Social Fund. The third author wishes to acknowledge financial support from the Polish National Science Centre, grant no. DEC-2011/01/B/ST6/07318.

\section{References}

[1] A. Andenmatten, Evaluation du risque de défaillance des émetteurs d'obligations. Une approche par l'aide multicritère à la décision, Collection Meta, Presses Polytechniques et Universitaires Romandes, 1995.

[2] K. Arrow, Social Choice and Individual Values, Yale University Press, 1951.

[3] K. Arrow, H. Raynaud, Social choice and multicriterion decision making, MIT Press, Cambridge, 1986.

[4] C. Barrett, P.K. Pattanaik, M. Salles, On choosing rationally when preferences are fuzzy, Fuzzy Sets and Systems 34 (1990) 197-212.

[5] N. Belnap, How a computer should think, in: Proc. of the Oxford International Symposium on Contemporary Aspects of Philosophy, pp. 30-56.

[6] N. Belnap, A useful four-valued logic, in: J.M. Dunn, G. Epstein (Eds.), Modern Uses of Multiple Valued Logics, D. Reidel, Dordrecht, 1977, pp. 8-37.

[7] R. Bisdorff, Logical foundation of fuzzy preferential systems with application to the electre decision aid methods, Computers \& Operations Research 27 (2000) 673-687.

[8] J. Błaszczyński, S. Greco, R. Słowiński, Inductive discovery of laws using monotonic rules, Engineering Applications of Artificial Intelligence 25 (2012) 284-294.

[9] J. Błaszczyński, S. Greco, R. Słowiński, M. Szeląg, On variable consistency dominance-based rough set approaches, in: S. Greco, Y. Hata, S. Hirano, M. Inuiguchi, S. Miyamoto, H.S. Nguyen, R. Słowiński (Eds.), Rough Sets and Current Trends in Computing 2006, volume 4259 of Lecture Notes in Artificial Intelligence, Springer, Berlin Heidelberg, 2006 , pp. 191-202.

[10] J. Błaszczyński, S. Greco, R. Słowiński, M. Szeląg, Monotonic variable consistency rough set approaches, in: J. Yao, P. Lingras, W. Wu, M. Szczuka, N.J. Cercone, D. Ślęzak (Eds.), Rough Sets and Knowledge Technology 2007, volume 4481 of Lecture Notes in Artificial Intelligence, Springer, Berlin Heidelberg, 2007, pp. 126-133.

[11] J. Błaszczyński, S. Greco, R. Słowiński, M. Szeląg, Monotonic variable consistency rough set approaches, International Journal of Approximate Reasoning 50 (2009) 979-999. doi:10.1016/j.ijar.2009.02.011.

[12] J. Błaszczyński, R. Słowiński, M. Szeląg, Probabilistic rough set approaches to ordinal classification with monotonicity constraints, in: E. Hüllermeier, R. Kruse, F. Hoffmann (Eds.), IPMU 2010, volume 6178 of Lecture Notes in Artificial Intelligence, Springer, Berlin Heidelberg, 2010, pp. 99-108.

[13] J. Błaszczyński, R. Słowiński, M. Szelag, Sequential covering rule induction algorithm for variable consistency rough set approaches, Information Sciences 181 (2011) 987-1002. doi:10.1016/j.ins.2010.10.030, available online 14 November 2010.

[14] D. Bouyssou, A note on the "min in favor" ranking method for valued preference relations, in: M. Cerny, D. Glükaufová, D. Loula (Eds.), Multicriteria Decision Making. Methods - Algorithms - Applications, Czechoslovak Academy of Sciences, Prague, 1992, pp. 16-25.

[15] D. Bouyssou, A note on the sum of differences choice function for fuzzy preference relations, Fuzzy Sets and Systems 47 (1992) 197-202.

[16] D. Bouyssou, Ranking methods based on valued preference relations: A characterization of the net flow method, European Journ. of Operational Research 60 (1992) 61-67.

[17] D. Bouyssou, A note on the 'min in favor' choice procedure for fuzzy preference relations, in: P. Pardalos, Y. Siskos, C. Zopounidis (Eds.), Advances in Multicriteria Analysis, Kluwer, 1995, pp. 9-16.

[18] D. Bouyssou, Outranking relations: do they have special properties?, Journal of Multi-Criteria Decision Analysis 5 (1996) 99-111.

[19] D. Bouyssou, Monotonicity of 'ranking by choosing': A progress report, Social Choice and Welfare 23 (2004) $249-273$. 
[20] D. Bouyssou, P. Perny, Ranking methods for valued preference relations: A characterization of a method based on leaving and entering flows, European Journal of Operational Research 61 (1992) 186-194.

[21] D. Bouyssou, M. Pirlot, Choosing and ranking on the basis of fuzzy preference relations with the "min in favour", in: G. Fandel, T. Gal (Eds.), Multiple Criteria Decision Making - Proceedings of the Twelfth International Conference Hagen (Germany), Springer, 1997, pp. 115-127.

[22] D. Bouyssou, P. Vincke, Ranking alternatives on the basis of preference relations: A progress report with special emphasis on outranking relations, Journal of Multi-Criteria Decision Analysis 6 (1997) 77-85.

[23] J. Brans, B. Mareschal, PROMETHEE methods, in: J. Figueira, S. Greco, M. Ehrgott (Eds.), Multiple Criteria Decision Analysis: State of the Art Surveys, Operations Research \& Management Science, Springer, New York, 2005, pp. $163-196$.

[24] J. Brans, B. Mareschal, P. Vincke, PROMETHEE: a new family of outranking methods in multicriteria analysis, in: J. Brans (Ed.), Operational Research, IFORS 84, North Holland, Amsterdam, 1984, pp. 477-490.

[25] K. Dembczyński, S. Greco, W. Kotłowski, R. Słowiński, Statistical model for rough set approach to multicriteria classification, in: J.N. Kok, J. Koronacki, R.L. de Mántaras, S. Matwin, D. Mladenič, A. Skowron (Eds.), Knowledge Discovery in Databases (PKDD 2007), volume 4702 of Lecture Notes in Artificial Intelligence, Springer, Warsaw, Poland, 2007, pp. $164-175$.

[26] K. Dembczyński, W. Kotłowski, R. Słowiński, Ensemble of decision rules for ordinal classification with monotonicity constraints, in: G. Wang, T. Li, J.W. Grzymała-Busse, D. Miao, A. Skowron, Y. Yao (Eds.), Rough Sets and Knowledge Technology 2008, volume 5009 of Lecture Notes in Artificial Intelligence, Springer, 2008, pp. $260-267$.

[27] K. Dembczyński, W. Kotłowski, R. Słowiński, Ordinal classification with decision rules, in: Z.W. Raś, S. Tsumoto, D. Zighed (Eds.), Mining Complex Data (MCD 2007), volume 4944 of Lecture Notes in Artificial Intelligence, Springer, 2008, pp. 169-181.

[28] K. Dembczyński, W. Kotłowski, R. Słowiński, M. Szeląg, Learning of rule ensembles for multiple attribute ranking problems, in: J. Fürnkranz, E. Hüllermeier (Eds.), Preference Learning, Springer, Berlin Heidelberg, 2010 , pp. $217-247$.

[29] K. Dembczyński, R. Pindur, R. Susmaga, Generation of exhaustive set of rules within dominance-based rough set approach, Electronic Notes in Theoretical Computer Science 82 (2003) 96-107.

[30] L.C. Dias, C. Lamboray, Extensions of the prudence principle to exploit a valued outranking relation, European Journal of Operational Research 201 (2010) 828-837. doi:10.1016/j.ejor.2009.03.026.

[31] J. Figueira, S. Greco, M. Ehrgott (Eds.), Multiple Criteria Decision Analysis: State of the Art Surveys, Operations Research \& Management Science, Springer, New York, 2005.

[32] J. Figueira, S. Greco, R. Słowiński, Building a set of additive value functions representing a reference preorder and intensities of preference: Grip method, European Journal of Operational Research 195 (2009) 460-486.

[33] J. Figueira, V. Mousseau, B. Roy, ELECTRE methods, in: J. Figueira, S. Greco, M. Ehrgott (Eds.), Multiple Criteria Decision Analysis: State of the Art Surveys, Operations Research \& Management Science, Springer, New York, 2005, pp. $133-162$.

[34] P. Fishburn, The Theory of Social Choice, Princeton University Press, 1973.

[35] P. Fortemps, S. Greco, R. Słowiński, Multicriteria decision support using rules that represent rough-graded preference relations, European Journal of Operational Research 188 (2008) 206-223.

[36] P. Fortemps, R. Słowiński, A graded quadrivalent logic for ordinal preference modelling: Loyola-like approach, Fuzzy Optimization and Decision Making 1 (2002) 93-111.

[37] E. Frank, M. Hall, A simple approach to ordinal classification, in: L. De Raedt, P.A. Flach (Eds.), Proc. of the European Conference on Machine Learning (ECML 2001), volume 2167 of Lecture Notes in Artificial Intelligence, Springer, Freiburg, Germany, 2001, pp. 145-157.

[38] Y. Freund, R. Iyer, R.E. Schapire, Y. Singer, An efficient boosting algorithm for combining preferences, Journal of Machine Learning Research 6 (2003) 933-969.

[39] J. Fürnkranz, E. Hüllermeier, Pairwise preference learning and ranking, in: N. Lavrac, D. Gamberger, L. Todorovski, H. Blockeel (Eds.), Proc. of the European Conference on Machine Learning (ECML 2003), volume 2837 of Lecture Notes in Artificial Intelligence, Springer, 2003, pp. 145-156.

[40] S. Giove, S. Greco, B. Matarazzo, R. Słowiński, Variable consistency monotonic decision trees, in: J.J. Alpigini, J.F. Peters, A. Skowron, N. Zhong (Eds.), Rough Sets and Current Trends in Computing (RSCTC 2002), volume 2475 of Lecture Notes in Artificial Intelligence, Springer, Malvern, USA, 2002, pp. 247-254.

[41] S. Greco, M. Kadziński, V. Mousseau, R. Słowiński, Electre ${ }^{G M S}$ : Robust ordinal regression for outranking methods, European Journal of Operational Research 214 (2011) 118-135.

[42] S. Greco, B. Matarazzo, R. Słowiński, Rough set approach to multi-attribute choice and ranking problems, Technical Report 38/95, ICS, Warsaw University of Technology, Warsaw, Poland, 1995.

[43] S. Greco, B. Matarazzo, R. Słowiński, Rough set approach to multi-attribute choice and ranking problems, in: G. Fandel, T. Gal (Eds.), Multiple Criteria Decision Making, Proceedings of the Twelfth International Conference, Hagen, Germany, Springer, Berlin, 1997, pp. 318-329.

[44] S. Greco, B. Matarazzo, R. Słowiński, Rough approximation of a preference relation by dominance relations, European Journal of Operational Research 117 (1999) 63-83.

[45] S. Greco, B. Matarazzo, R. Słowiński, The use of rough sets and fuzzy sets in MCDM, in: T. Gal, T.J. Stewart, T. Hanne (Eds.), Multicriteria Decision Making: Advances in MCDM models, Algorithms, Theory, and Applications, volume 21 of International Series in Operations Research \& Management Science, Kluwer, Dordrecht, 1999, pp. 14.1-14.59.

[46] S. Greco, B. Matarazzo, R. Słowiński, Rough sets theory for multicriteria decision analysis, European Journal of Operational Research 129 (2001) 1-47.

[47] S. Greco, B. Matarazzo, R. Słowiński, Axiomatic characterization of a general utility function and its particular cases 
in terms of conjoint measurement and rough-set decision rules, European Journal of Operational Research 158 (2004) 271-292.

[48] S. Greco, B. Matarazzo, R. Słowiński, Decision rule approach, in: J. Figueira, S. Greco, M. Ehrgott (Eds.), Multiple Criteria Decision Analysis: State of the Art Surveys, Operations Research \& Management Science, Springer, New York, 2005, pp. 507-562.

[49] S. Greco, B. Matarazzo, R. Słowiński, Preference representation by means of conjoint measurement and decision rule model, in: D. Bouyssou, E. Jacquet-Lagrèze, P. Perny, R. Słowiński, D. Vanderpooten, P. Vincke (Eds.), Aiding Decisions with Multiple Criteria - Essays in Honor of Bernard Roy, Kluwer, Boston, 2005, pp. 263-313.

[50] S. Greco, B. Matarazzo, R. Słowiński, Dominance-based rough set approach as a proper way of handling graduality in rough set theory, in: Transactions on Rough Sets VII, volume 4400 of LNCS, Springer, Berlin, 2007 , pp. 36-52.

[51] S. Greco, B. Matarazzo, R. Słowiński, Dominance-based rough set approach to interactive multiobjective optimization, in: J. Branke, K. Deb, K. Miettinen, R. Słowiński (Eds.), Multiobjective Optimization: Interactive and Evolutionary Approaches, volume 5252 of Lecture Notes in Computer Science, Springer, Berlin, 2008, pp. 121-156.

[52] S. Greco, B. Matarazzo, R. Słowiński, Dominance-based rough set approach to decision under uncertainty and time preference, Annals of Operations Research 176 (2010) 41-75. doi:10.1007/s10479-009-0566-8.

[53] S. Greco, B. Matarazzo, R. Słowiński, A. Tsoukiàs, Exploitation of a rough approximation of the outranking relation in multicriteria choice and ranking, in: T.J. Stewart, R.C. van den Honert (Eds.), Trends in Multicriteria Decision Making, volume 465 of Lecture Notes in Economics and Mathematical Systems, Springer, Berlin, 1998, pp. 45-60.

[54] S. Greco, V. Mousseau, R. Słowiński, Ordinal regression revisited: multiple criteria ranking using a set of additive value functions, European Journal of Operational Research 191 (2008) 415-435.

[55] S. Greco, Z. Pawlak, R. Słowiński, Can bayesian confirmation measures be useful for rough set decision rules?, Engineering Applications of Artificial Intelligence 17 (2004) 345-361.

[56] S. Greco, R. Słowiński, J. Figueira, V. Mousseau, Robust ordinal regression, in: M. Ehrgott, J. Figueira, S. Greco (Eds.), Trends in Multiple Criteria Decision Analysis, International Series in Operations Research \& Management Science, Springer, New York, 2010, pp. 241-283.

[57] S. Greco, R. Słowiński, B. Matarazzo, An algorithm for induction of decision rules consistent with dominance principle, in: W. Ziarko, Y.Y. Yao (Eds.), Rough Sets and Current Trends in Computing 2001, volume 2005 of Lecture Notes in Artificial Intelligence, Springer, Berlin, 2001, pp. 304-313.

[58] S. Greco, R. Słowiński, B. Matarazzo, J. Stefanowski, Variable consistency model of dominance-based rough sets approach, in: W. Ziarko, Y.Y. Yao (Eds.), Rough Sets and Current Trends in Computing 2001, volume 2005 of Lecture Notes in Artificial Intelligence, Springer, Berlin, 2001, pp. 170-181.

[59] S. Greco, R. Słowiński, I. Szczęch, Properties of rule interestingness measures and alternative approaches to normalization of measures, Information Sciences 216 (2012) 1-16.

[60] D. Henriet, The copeland choice function - an axiomatic characterization, Social Choice and Welfare 2 (1985) $49-64$.

[61] R. Herbrich, T. Graepel, K. Obermayer, Regression Models for Ordinal Data: A Machine Learning Approach, Technical report TR-99/03, TU Berlin, 1999.

[62] W. Kotłowski, K. Dembczyński, S. Greco, R. Słowiński, Stochastic dominance-based rough set model for ordinal classification, Information Sciences 178 (2008) 4019-4037.

[63] P. Langley, H.A. Simon, Fielded applications of machine learning, in: R.S. Michalski, I. Bratko, M. Kubat (Eds.), Machine Learning and Data Mining, Wiley, New York, 1998, pp. 113-129.

[64] J.G. March, Bounded rationality, ambiguity, and the engineering of choice, in: D.E. Bell, H. Raiffa, A. Tversky (Eds.), Decision Making, Descriptive, Normative and Prescriptive Interactions, Cambridge University Press, New York, 1988, pp. $33-58$.

[65] R.S. Michalski, A theory and methodology of inductive learning, in: R.S. Michalski, J.G. Carbonell, T.M. Mitchell (Eds.), Machine Learning: An Artificial Intelligence Approach, Tioga Publishing, Palo Alto, 1983, pp. 83-129.

[66] V. Mousseau, R. Słowiński, Inferring an ELECTRE TRI model from assignment examples, Journal of Global Optimization 12 (1998) 157-174.

[67] Z. Pawlak, Rough Sets. Theoretical Aspects of Reasoning about Data, Kluwer, Dordrecht, Netherlands, 1991.

[68] Z. Pawlak, Rough sets and intelligent data analysis, Information Sciences 147 (2002) 1-12.

[69] Z. Pawlak, A. Skowron, Rudiments of rough sets, Information Sciences 177 (2007) 3-27.

[70] Z. Pawlak, R. Słowiński, Rough set approach to multi-attribute decision analysis, European Journal of Operational Research 72 (1994) 443-459.

[71] P. Perny, B. Roy, The use of fuzzy outranking relations in preference modelling, Fuzzy Sets and Systems 49 (1992) $33-53$.

[72] P. Perny, A. Tsoukias, On the continuous extension of a four valued logic for preference modelling, in: Proceedings of the IPMU 98 conference, pp. 302-309.

[73] M. Pirlot, A characterization of 'min' as a procedure for exploiting valued preference relations and related results, Journal of Multi-Criteria Decision Analysis 4 (1995) 37-56.

[74] J. Rennie, N. Srebro, Loss functions for preference levels: Regression with discrete ordered labels, in: Proc. of the IJCAI Multidisciplinary Workshop on Advances in Preference Handling, Edinburgh, Scotland.

[75] B. Roy, ELECTRE III : Un algorithme de classements fondé sur une représentation floue des préférences en présence de critères multiples, Cahiers du CERO 20 (1978) 3-24.

[76] B. Roy, The outranking approach and the foundation of ELECTRE methods, Theory and Decision 31 (1991) $49-73$.

[77] B. Roy, Multicriteria Methodology for Decision Aiding, Kluwer, Dordrecht, 1996.

[78] B. Roy, D. Bouyssou, Aide Multicritère à la Décision: Méthodes et Cas, Economica, Paris, 1993.

[79] A. Rubinstein, Ranking the participants in a tournament, SIAM J. of Applied Mathematics 38 (1980) 108-111. 
[80] P.J.H. Shoemaker, The expected utility model: its variants, purposes, evidence and limitations, Journal of Economic Literature 20 (1982) 529-562.

[81] P. Slovic, Choice between equally-valued alternatives, Journal of Experimental Psychology: Human Perception Performance 1 (1975) 280-287.

[82] R. Słowiński, Rough set learning of preferential attitude in multi-criteria decision making, in: J. Komorowski, Z. Raś (Eds.), Methodologies for Intelligent Systems, volume 689 of Lecture Notes in Artificial Intelligence, Springer, Berlin, 1993, pp. 642-651.

[83] R. Słowiński, S. Greco, B. Matarazzo, Axiomatization of utility, outranking and decision-rule preference models for multiple-criteria classification problems under partial inconsistency with the dominance principle, Control \& Cybernetics 31 (2002) 1005-1035.

[84] R. Słowiński, S. Greco, B. Matarazzo, Rough sets in decision making, in: R. Meyers (Ed.), Encyclopedia of Complexity and Systems Science, Springer, New York, 2009, pp. 7753-7786.

[85] R. Słowiński, S. Greco, B. Matarazzo, Rough set based decision support, in: E.K. Burke, G. Kendall (Eds.), Search Methodologies: Introductory Tutorials in Optimization and Decision Support Techniques, 2nd ed., Springer, New York, 2014, pp. 557-609.

[86] N. Srebro, J. Rennie, T. Jaakkola, Maximum margin matrix factorizations, in: Advances in Neural Information Processing Systems 17 (NIPS 2004), MIT Press, 2005, pp. 1329-1336.

[87] M. Szeląg, S. Greco, R. Słowiński, Rule-based approach to multicriteria ranking, in: M. Doumpos, E. Grigoroudis (Eds.), Multicriteria Decision Aid and Artificial Intelligence: Links, Theory and Applications, Wiley, 2013, pp. 127-160.

[88] M. Szeląg, R. Słowiński, J. Błaszczyński, jRank - ranking using dominance-based rough set approach, Newsletter of the European Working Group "Multiple Criteria Decision Aiding" 3(22) (2010) 13-15.

[89] A. Tsoukias, P. Vincke, A new axiomatic foundation of partial comparability, Theory and Decision 39 (1995) $79-114$.

[90] P. Vincke, Exploitation of a crisp relation in a ranking problem, Theory and Decision 32 (1992) $221-240$.

[91] H. Young, An axiomatization of Borda's rule, Journ. of Economic Theory 9 (1974) 43-52.

\section{Appendix}

Definition 13 (Monotonicity property (m1)). A cost-type consistency measure $\Theta_{T}, T \in\left\{S, S^{c}\right\}$, has monotonicity property $(\mathrm{m} 1)$ iff it is monotonically non-increasing w.r.t. the considered set of criteria, i.e., iff for all $P \subseteq R \subseteq G$, and for all $(a, b) \in B$

$$
\Theta_{T}^{P}(a, b) \geq \Theta_{T}^{R}(a, b)
$$

where $\Theta_{T}^{P}(a, b)$ denotes the value of measure $\Theta_{T}$ calculated for pair of objects $(a, b)$ taking into account only criteria from set $P \subseteq G$.

Definition 14 (Monotonicity property (m2)). A cost-type consistency measure $\Theta_{T}, T \in\left\{S, S^{c}\right\}$, has monotonicity property $(\mathrm{m} 2)$ iff it is monotonically non-increasing w.r.t. the considered comprehensive preference relation $T$, i.e., iff for all $T^{\prime}=T \cup T^{\Delta}, T^{\Delta} \cap B=\emptyset$, and for all $(a, b) \in B$

$$
\Theta_{T}(a, b) \geq \Theta_{T^{\prime}}(a, b) \text {. }
$$

Definition 15 (Monotonicity property (m4)). A cost-type consistency measure $\Theta_{T}, T \in\left\{S, S^{c}\right\}$, has monotonicity property $(\mathrm{m} 4)$ iff it is monotonically non-increasing w.r.t. dominance relation $D_{2}$ over $B$, i.e., iff

$$
\forall(a, b),(c, d) \in B:(a, b) D_{2}(c, d) \Rightarrow \Theta_{T}(a, b) \leq \Theta_{T}(c, d)
$$

Proof (Corollary 1). Let us consider any two objects $a, b \in A$, such that $a D b$, and let us denote by $D_{2}^{\prime}$ the dominance relation over set $A \times A$, defined in the same way as the dominance relation $D_{2}$ over set $B$, with the only difference that $B$ (appearing in the definition of $D_{2}$ ) is replaced by $A \times A$. First, let us observe that $a D b$ implies that $(a, b) D_{2}^{\prime}(b, a)$, and, moreover, given any object $c \in A \backslash\{a, b\}$, it is true that $(a, c) D_{2}^{\prime}(b, c)$ and $(c, b) D_{2}^{\prime}(c, a)$. Secondly, note that every decision rule $r_{S} \in R_{S}$ that covers the dominated (w.r.t. $D_{2}^{\prime}$ ) pair of objects $(b, a)$ (respectively, $(b, c),(c, a)$ ), covers also the dominating (w.r.t. $\left.D_{2}^{\prime}\right)$ pair of objects $(a, b)$ (respectively, $(a, c),(c, b))$. Analogously, every decision rule $r_{S^{c}} \in R_{S^{c}}$ that covers the dominating pair of objects $(a, b)$ (respectively, $(a, c),(c, b)$ ), covers also the dominated pair of objects $(b, a)$ (respectively, $(b, c)$, $(c, a))$. Therefore, after application of decision rules on set $A$, according to definitions (22) and (23) we get:

- $\mathcal{S}(a, b) \geq \mathcal{S}(b, a)$ and $\mathcal{S}^{c}(b, a) \geq \mathcal{S}^{c}(a, b)$, 
- $\mathcal{S}(a, c) \geq \mathcal{S}(b, c)$ and $\mathcal{S}^{c}(c, a) \geq \mathcal{S}^{c}(c, b)$,

- $\mathcal{S}(c, a) \leq \mathcal{S}(c, b)$ and $\mathcal{S}^{c}(a, c) \leq \mathcal{S}^{c}(b, c)$.

Thirdly, from (29), we get $\mathcal{R}(a, b) \geq \mathcal{R}(b, a)$, and, moreover, $\mathcal{R}(a, c) \geq \mathcal{R}(b, c)$, and $\mathcal{R}(c, a) \leq \mathcal{R}(c, b)$. This set of inequalities is the antecedent of the implication given in Definition 3 of property $C C$. Thus, from this definition, we have $a \succeq(A, \mathcal{R}) b$.

Proof (Properties of NFR).

$(N)$ Satisfied according to Bouyssou [16], Bouyssou and Vincke [22].

(M) Satisfied due to the definition of NFR, given by (41).

$(C C)$ According to Bouyssou and Vincke [22], this property is satisfied in case of exploitation of a crisp relation. However, it is evident that this property is also satisfied in general, i.e., when an exploited relation is fuzzy.

(INDO) According to Bouyssou and Vincke [22], this property is satisfied in case of exploitation of a crisp relation. However, it is evident that this property is also satisfied in general, i.e., when an exploited relation is fuzzy.

(IC) Satisfied according to Bouyssou [16], Bouyssou and Vincke [22].

$(O)$ Not satisfied since for a given finite set of objects $A$ and for a fuzzy relation $R$ over $A, N F R$ makes use of the "cardinal" properties of values $R(a, b)$, with $a, b \in A[22]$.

(C) Satisfied according to Bouyssou and Vincke [22].

$(F)$ Satisfied according to Bouyssou and Vincke [22].

$(D P)$ Satisfied according to Bouyssou and Vincke [22].

$(G F)$ Not satisfied according to Bouyssou and Vincke [22].

Proof (Properties of It.NFR).

$(N)$ According to Bouyssou and Vincke [22], this property is satisfied in case of exploitation of a crisp relation. However, it is evident that this property is also satisfied in general, i.e., when an exploited relation is fuzzy.

$(M)$ Not satisfied, as shown by the following example. Consider set $A=\{a, b, c, d, e, f\}$, and fuzzy relation $R$ over $A$ defined as: $R(a, d)=0.5, R(b, c)=0.5, R(c, a)=1, R(c, e)=1, R(d, b)=1, R(d, f)=0.5$, $R(x, x)=1$ for all $x \in A$, and $R(x, y)=0$ for the remaining pairs $(x, y) \in A \times A$. The ranking (weak order) obtained for relation $R$ is: $c \succ d \succ a, b, e, f$ (i.e., object $c$ is the best, object $d$ is second best, and the remaining objects are in the third equivalence class). Observe that we have $a \succeq(A, R) b$. Now, consider relation $R^{\prime}$ which is identical to $R$ except that $R^{\prime}(a, c)=1$. Thus, object $a$ is improved. However, the ranking (weak order) obtained for relation $R^{\prime}$ is: $d \succ b, c \succ a, e, f$, i.e., it is not true that $a \succeq\left(A, R^{\prime}\right) b$.

$(C C)$ According to Bouyssou and Vincke [22], this property is satisfied in case of exploitation of a crisp relation. However, it is evident that this property is also satisfied in general, i.e., when an exploited relation is fuzzy.

(INDO) According to Bouyssou and Vincke [22], this property is satisfied in case of exploitation of a crisp relation. However, it is evident that this property is also satisfied in general, i.e., when an exploited relation is fuzzy. 
(IC) Not satisfied, as shown by the following example. Consider set $A=\{a, b, c, d, e\}$, and two fuzzy relations $R, R^{\prime}$ over $A$ defined as:

- $R(a, b)=1, R(b, c)=1, R(c, a)=1, R(b, e)=0.5, R(c, d)=1, R(x, x)=1$ for all $x \in A$, and $R(x, y)=0$ for the remaining pairs $(x, y) \in A \times A$,

- $R^{\prime}(b, e)=0.5, R^{\prime}(c, d)=1, R^{\prime}(x, x)=1$ for all $x \in A$, and $R^{\prime}(x, y)=0$ for the remaining pairs $(x, y) \in A \times A$.

Thus, $R$ and $R^{\prime}$ are circuit-equivalent ( $R^{\prime}$ is identical to $R$ except for the circuit $a-b-c$ of length 3 , on which value $\epsilon=1$ has been subtracted). If property $I C$ would be satisfied, we would have $\succeq\left(A, R^{\prime}\right)=\succeq(A, R)$. However, the ranking (weak order) obtained for relation $R$ is: $c \succ a \succ b \succ e, d$ (i.e., object $c$ is the best, object $a$ is second best, object $b$ is third best, and the remaining objects are in the fourth equivalence class), while the ranking (weak order) obtained for relation $R^{\prime}$ is: $c \succ b \succ a, e, d$. Thus, we obtain that $\succeq\left(A, R^{\prime}\right) \neq \succeq(A, R)$.

$(O)$ Not satisfied since for a given finite set of objects $A$ and for a fuzzy relation $R$ over $A, I t . N F R$ makes use of the "cardinal" properties of values $R(a, b)$, with $a, b \in A$.

(C) Not satisfied, as shown by the following example. Consider set $A=\{a, b, c, d\}$, and the family of fuzzy relations $R^{\epsilon}$ over $A$, with $\epsilon \in(0,1]$, defined by Table 9 . For any $\epsilon \in(0,1]$, we have $c \succeq\left(A, R^{\epsilon}\right) d$ (as

\begin{tabular}{|c|c|c|c|c|}
\hline$R^{\epsilon}$ & $a$ & $b$ & $c$ & $d$ \\
\hline$a$ & - & 1 & 1 & 0 \\
\hline$b$ & 1 & - & 0 & 0 \\
\hline$c$ & 0 & 1 & - & 1 \\
\hline$d$ & 0 & $1-\epsilon$ & 1 & - \\
\hline
\end{tabular}

object $a$ is always chosen in the first iteration, and object $c$ in the second iteration), while for relation $R$ (obtained when $\epsilon=0$ ), we have $d \succ(A, R) c$ (as objects $a$ and $d$ are chosen in the first iteration), which violates continuity.

$(F)$ Satisfied according to Bouyssou and Vincke [22].

$(D P)$ Satisfied according to Bouyssou and Vincke [22].

$(G F)$ Not satisfied since $N F R$ does not satisfy $G F$, and the first equivalence classes of the weak orders produced by NFR and It.NFR are the same.

Proof (Properties of MiF).

$(N)$ Satisfied according to Bouyssou [14], Pirlot [73], Bouyssou and Vincke [22].

$(M)$ Satisfied. Note that given a finite set of objects $A$ and a fuzzy relation $R$ over this set, objects from $A$ are ranked according to their scores calculated by function $m F$ (33). Thus, we have $a \succeq(A, R) b \Leftrightarrow$ $m F(a, A, R) \geq m F(b, A, R)$. Then, if the value $R(a, c)$, for some $c \in A \backslash\{a\}$, is improved, the score of object $a$ cannot decrease; the change of value $R(c, a)$, for some $c \in A \backslash\{a\}$ does not affect the score of object $a$. Moreover, if the value $R(b, d)$, for some $d \in A \backslash\{b\}$, is decreased, the score of object $b$ cannot increase; the change of value $R(d, b)$, for some $d \in A \backslash\{a\}$, does not affect the score of object $b$. Thus, for any of the four considered changes of relation $R$, reflected by relation $R^{\prime}$, we have $\left(a \succeq(A, R) b \Rightarrow a \succeq\left(A, R^{\prime}\right) b\right)$. 
$(C C)$ Satisfied. Note that given a finite set of objects $A$ and a fuzzy relation $R$ over this set, objects from $A$ are ranked according to their scores calculated by function $m F(33)$. If $R(a, b) \geq R(b, a)$ and for all $c \in A \backslash\{a, b\}$ there is $R(a, c) \geq R(b, c)$, then according to definition (33), we have $m F(a, A, R) \geq$ $m F(b, A, R)$. It implies that $a \succeq(A, R) b$.

(INDO) Not satisfied, as shown by the following example. Consider set $A=\{a, b, c, d\}$, and fuzzy relation $R$ over $A$ defined as: $R(a, b)=1, R(a, c)=1, R(a, d)=0.5, R(b, a)=1, R(b, c)=0.5, R(b, d)=0.5$, $R(c, d)=0.5, R(x, x)=1$ for all $x \in A$, and $R(x, y)=0$ for the remaining pairs $(x, y) \in A \times A$. Observe that object $d$ is a non-discriminating object since $R(x, d)=0.5$ and $R(d, x)=0$, for $x \in A^{\prime}$, with $A^{\prime}=\{a, b, c\}$. We obtain $\succeq\left(A^{\prime}, R / A^{\prime}\right)=a \succ b \succ c$ (i.e., object $a$ is the best, and object $b$ is better than object $c$ ). This ranking is different than $\succeq(A, R) / A^{\prime}=a, b, c$ (i.e., all three objects are in the first equivalence class).

(IC) Not satisfied according to Bouyssou and Vincke [22].

(O) Satisfied according to Bouyssou [14], Pirlot [73], Bouyssou and Vincke [22], Bouyssou and Pirlot [21].

(C) Satisfied according to Bouyssou [14], Bouyssou and Vincke [22], Bouyssou and Pirlot [21].

(F) Not satisfied according to Bouyssou and Vincke [22], Bouyssou and Pirlot [21].

$(D P)$ Satisfied. Due to transitivity of a crisp relation $R$ over a given finite set of objects $A$, for any pair of objects $(a, b) \in R$ we have $m F(a, A, R) \geq m F(b, A, R)$. This implies that $a \succeq(A, R) b$. Thus, $R \subseteq \succeq(A, R)$.

(GF) Satisfied according to Bouyssou and Vincke [22], Bouyssou and Pirlot [21].

Proof (Properties of It.MiF).

$(N)$ Obviously satisfied.

(M) Not satisfied, as shown by the following example. Consider set $A=\{a, b, c\}$, and fuzzy relation $R$ over $A$ defined as: $R(a, b)=0.5, R(b, a)=0.5, R(c, a)=1, R(c, b)=1, R(b, c)=0.5, R(x, x)=1$ for all $x \in A$, and $R(x, y)=0$ for the remaining pairs $(x, y) \in A \times A$. The ranking (weak order) obtained for relation $R$ is: $c \succ a, b$ (i.e., object $c$ is the best, and objects $a$ and $b$ are in the second equivalence class). Observe that we have $a \succeq(A, R) b$. Now, consider relation $R^{\prime}$ which is identical to $R$ except for the pair of objects $(c, a)$, for which we have lower value $R^{\prime}(c, a)=0$. This difference between $R$ and $R^{\prime}$ should not "negatively affect" object $a$. However, the ranking (weak order) obtained for relation $R^{\prime}$ is: $b \succ a, c$. Thus, it is not true that $a \succeq\left(A, R^{\prime}\right) b$.

$(C C)$ Satisfied. Observe that, given a finite set of objects $A$ and a fuzzy relation $R$ over $A$, if object $a$ "covers" object $b$ w.r.t. set $A$, it is also true that object $a$ "covers" object $b$ w.r.t. any subset $A^{\prime} \subseteq A$. Using the reasoning from the proof of property $C C$ of the $M i F$ ranking method, this implies that for any $A^{\prime} \subseteq A, m F\left(a, \mathcal{A}^{\prime}, R\right) \geq m F\left(b, \mathcal{A}^{\prime}, R\right)$. Thus, in any iteration where both objects $a$ and $b$ are considered (i.e., $a, b \in A^{i}$, with $A^{i} \subseteq A$ ), it is impossible (since the choice among objects belonging to set $A^{i}$ is made based on maximum score, and the score of each object $c \in A^{i}$ is given by $m F\left(c, A^{i}, R\right)$ ) that object $b$ will we chosen while object $a$ will not be chosen. Thus, $a \succeq(A, R) b$.

(INDO) Not satisfied. Consider the set $A$ and fuzzy relation $R$ given in the proof of property $I N D O$ of the $M i F$ ranking method. We obtain $\succeq\left(A^{\prime}, R / A^{\prime}\right)=a \succ b \succ c$ (i.e., object $a$ is the best, and object $b$ is better than object $c$ ). This ranking is different than $\succeq(A, R) / A^{\prime}=a, b, c$ (i.e., all three objects are in the first equivalence class).

(IC) Not satisfied since $M i F$ does not satisfy $I C$, and the first equivalence classes of the weak orders produced by $M i F$ and $I t . M i F$ are the same. 
(O) Satisfied according to Bouyssou and Pirlot [21].

(C) Not satisfied according to Bouyssou and Pirlot [21].

$(F)$ Satisfied according to Bouyssou and Pirlot [21].

$(D P)$ Satisfied. Let us consider a finite set of objects $A$, a transitive and crisp relation $R$ over $A$, and any pair of objects $(a, b) \in R$. Observe that due to transitivity of $R$, relation $R / A^{\prime}$ is also transitive, for any $A^{\prime} \subseteq A$. This implies that in each $i$-th iteration, where a choice is made among objects belonging to set $A^{i} \subseteq A$, if the pair of objects $(a, b)$ belongs to $R / A^{i}$, it is true that $m F\left(a, A^{i}, R\right) \geq m F\left(b, A^{i}, R\right)$. Thus, it is impossible (since the choice among objects belonging to set $A^{i}$ is made based on maximum score, and the score of each object $c \in A^{i}$ is given by $\left.m F\left(c, A^{i}, R\right)\right)$ that object $b$ will we chosen while object $a$ will not be chosen. Therefore, after all iterations, we have to obtain that $a \succeq(A, R) b$. This implies that $R \subseteq \succeq(A, R)$.

$(G F)$ Satisfied according to Bouyssou and Pirlot [21].

Proof (Properties of $L / E$ ).

$(N)$ Obviously satisfied.

(M) Satisfied according to Bouyssou and Perny [20].

$(C C)$ Satisfied. Let us consider a finite set of objects $A$ and a fuzzy relation $R$ over $A$. If $R(a, b) \geq R(b, a)$ and for all $c \in A \backslash\{a, b\}$ there is $R(a, c) \geq R(b, c)$ and $R(c, a) \leq R(c, b)$, then according to definitions (34) and (37), we have $S F(a, A, R) \geq S F(b, A, R)$ (in other words, object $a$ has not smaller leaving flow than object $b$ ) and $-S A(a, A, R) \geq-S A(b, A, R)$ (in other words, object $a$ has not greater entering flow than object $b$ ). Due to the definition of $L / E$ (45), it implies that $a \succeq(A, R) b$.

(INDO) Satisfied. Let us consider a finite set of objects $A$ and a fuzzy relation $R$ over $A$. Then, each nondiscriminating object $b \in A \backslash A^{\prime}$, with $A^{\prime} \subset A$, influences leaving and entering flow of each object $a \in A^{\prime}$ in the same way. Precisely, leaving flow $S F\left(a, A^{\prime}, R\right)$ of each object $a \in A^{\prime}$ increases (or decreases) by $k$ while entering flow $-\left(-S A\left(a, A^{\prime}, R\right)\right)$ of each object $a \in A^{\prime}$ increases (or decreases) by $k^{\prime}$.

(IC) Not satisfied, as shown by the following example. Consider set $A=\{a, b, c, d, e, f\}$, and two fuzzy relations $R, R^{\prime}$ over $A$, defined as:

- $R(a, b)=0.5, R(b, c)=0.5, R(c, a)=0.5, R(e, d)=0.5, R(d, f)=0.5, R(x, x)=1$ for all $x \in A$, and $R(x, y)=0$ for the remaining pairs $(x, y) \in A \times A$,

- $R^{\prime}(a, b)=1, R^{\prime}(b, c)=1, R^{\prime}(c, a)=1, R^{\prime}(e, d)=0.5, R^{\prime}(d, f)=0.5, R^{\prime}(x, x)=1$ for all $x \in A$, and $R^{\prime}(x, y)=0$ for the remaining pairs $(x, y) \in A \times A$.

Thus, $R$ and $R^{\prime}$ are circuit-equivalent ( $R^{\prime}$ is identical to $R$ except for the circuit $a-b-c$ of length 3 , on which value $\epsilon=0.5$ has been added). If property $I C$ would be satisfied, we would have $\succeq\left(A, R^{\prime}\right)=\succeq$ $(A, R)$. However, we have $d \succeq(A, R) b$ and $b \succeq(A, R) d$ (i.e., objects $b$ and $d$ are in the same equivalence class in case of exploitation of relation $R$ ), while not $d \succeq\left(A, R^{\prime}\right) b$ nor $b \succeq\left(A, R^{\prime}\right) d$ (i.e., objects $b$ and $d$ are incomparable in case of exploitation of relation $\left.R^{\prime}\right)$. Thus, we obtain that $\succeq\left(A, R^{\prime}\right) \neq \succeq(A, R)$.

$(O)$ Not satisfied since for a given finite set of objects $A$ and for a fuzzy relation $R$ over $A, L / E$ makes use of the "cardinal" properties of values $R(a, b)$, with $a, b \in A$.

(C) Obviously satisfied. 
$(F)$ Satisfied. Let us consider a finite set of objects $A$ and a weak order relation $R$ over $A$. First, due to transitivity of $R$, given any pair of objects $(a, b) \in R$, object $a$ has not smaller leaving flow and not greater entering flow than object $b$, i.e., $S F(a, A, R) \geq S F(b, A, R)$ and $-S A(a, A, R) \geq-S A(b, A, R)$, respectively. Thus, $a \succeq(A, R) b$. This means that $R \subseteq \succeq(A, R)$. Second, due to transitivity and completeness of $R$, given any pair of objects $(a, b) \notin R$, object $a$ has smaller leaving flow and greater entering flow than object $b$. Therefore, it is not true that $a \succeq(A, R) b$. This means that $\neg R \subseteq \neg \succeq$ $(A, R)$, where $\neg$ denotes complement of a set. Thus, $\succeq(A, R)=R$.

$(D P)$ Satisfied as shown in the first part of the proof of property $F$ above.

$(G F)$ Satisfied. Let us consider a finite set of objects $A$ and a crisp relation $R$ over $A$. First, assume that the antecedent of the implication in the definition of property $G F$ is true. Thus, $G(A, R) \neq \emptyset$. Second, due to the definition of $L / E$, given by (45), every object $a \in G(A, \succeq(A, R))$ has maximum leaving flow and minimum entering flow among all objects from set $A$. To have maximum leaving flow, each object $a \in G(A, \succeq(A, R))$ has to belong to set $G(A, R)$. 
Table 10: Evaluations on criteria and category assignments of all airline-year objects considered in the Illustrative Example (Section 9)

\begin{tabular}{|c|c|c|c|c|c|c|c|c|c|c|c|c|}
\hline Id & Airline-year & $g_{1}$ & $g_{2}$ & $g_{3}$ & $g_{4}$ & $g_{5}$ & $g_{6}$ & $g_{7}$ & $g_{8}$ & $g_{9}$ & $g_{10}$ & Cat. \\
\hline$a_{1}$ & japan-89 & 14.01 & 5.06 & 4.7 & 31.23 & 16.8 & 38.55 & 11.73 & 1.3 & 45.7 & 22.1 & $U I G$ \\
\hline$a_{2}$ & japan-90 & 13.93 & 5.09 & 6.3 & 38.46 & 18.4 & 4.02 & 2.55 & 15.3 & 67.9 & 25.1 & $U I G$ \\
\hline$a_{3}$ & japan-91 & 13.59 & 5.03 & 7.3 & 44.4 & 19.7 & 7.22 & 3.68 & 9.2 & 58.5 & 23 & $U I G$ \\
\hline$a_{4}$ & japan-92 & 12.93 & 4.92 & 8.6 & 48.78 & 21 & 6.64 & 3.2 & 11 & 45.9 & 20.2 & $U I G$ \\
\hline$a_{5}$ & amr-88 & 12.42 & 4.93 & 3.1 & 59.36 & 32 & 17.47 & 6.47 & 1.9 & 50.2 & 32.4 & $L I G$ \\
\hline$a_{6}$ & amr-89 & 11.95 & 4.85 & 3.2 & 54.97 & 33.2 & 8.98 & 4.1 & 2.9 & 48.2 & 34.6 & $L I G$ \\
\hline$a_{7}$ & amr-90 & 10.92 & 4.33 & 3.7 & 48.23 & 32 & 7.95 & 3.05 & 4.9 & 40.7 & 27.9 & $L I G$ \\
\hline$a_{8}$ & amr-91 & 10.48 & 4.04 & 4.1 & 44.28 & 30 & 7.86 & 2.52 & 6.8 & 34.3 & 23.4 & $L I G$ \\
\hline$a_{9}$ & amr-92 & 9.65 & 3.66 & 5.3 & 39.76 & 27.2 & 5.97 & 2.14 & 9.9 & 25.4 & 17.9 & $L I G$ \\
\hline$a_{10}$ & ba-93 & 8.98 & 4.09 & 4.4 & 32.72 & 22.2 & 9.29 & 3.56 & 6.4 & 28.7 & 19.1 & $L I G$ \\
\hline$a_{11}$ & delta-89 & 12.2 & 7.51 & 1.3 & 52.41 & 37 & 13.94 & 12.01 & 0.6 & 58.5 & 40.4 & $L I G$ \\
\hline$a_{12}$ & delta-90 & 10.89 & 7.14 & 1.6 & 54.5 & 37.1 & 6.15 & 4.74 & 2.7 & 53.6 & 35.9 & $L I G$ \\
\hline$a_{13}$ & delta-91 & 9.96 & 6.71 & 2.3 & 54.91 & 36 & 3.94 & 2.59 & 5.9 & 43.6 & 29.2 & $L I G$ \\
\hline$a_{14}$ & delta-92 & 7.69 & 5.73 & 5.3 & 49.7 & 35.2 & 2.05 & 1.76 & 16.5 & 26.7 & 18.6 & $L I G$ \\
\hline$a_{15}$ & delta-93 & 6.91 & 5.1 & 5.9 & 41.83 & 28.2 & 8.47 & 4.38 & 3.7 & 26.8 & 16.8 & $L I G$ \\
\hline$a_{16}$ & japan-93 & 10.83 & 4.31 & 20 & 50.67 & 21.3 & -2.31 & 0.37 & 20 & 35.4 & 15.9 & $L I G$ \\
\hline$a_{17}$ & nwa-88 & 13.93 & 14.09 & 2.3 & 53.37 & 38.2 & 12.75 & 8.96 & 1.3 & 49.4 & 37.4 & $L I G$ \\
\hline$a_{18}$ & stw-89 & 16.34 & 4.37 & 2.7 & 54.82 & 45.4 & 15.81 & 4.67 & 2.3 & 48.8 & 41.5 & $L I G$ \\
\hline$a_{19}$ & stw-88 & 15.77 & 4.29 & 2.7 & 57.5 & 48.3 & 19.49 & 5.36 & 2.3 & 54.8 & 43.4 & $L I G$ \\
\hline$a_{20}$ & stw-90 & 15.77 & 4.42 & 2.4 & 53.48 & 44.6 & 12.96 & 4.36 & 2.2 & 46.2 & 41.1 & $L I G$ \\
\hline$a_{21}$ & stw-91 & 14.48 & 4.29 & 2.8 & 50.83 & 41.9 & 9.8 & 3.17 & 4.8 & 42.1 & 34.2 & $L I G$ \\
\hline$a_{22}$ & stw-92 & 14.76 & 4.43 & 2.9 & 47.93 & 39.5 & 15.75 & 4.57 & 2.7 & 47.9 & 37.3 & $L I G$ \\
\hline$a_{23}$ & united-89 & 8.02 & 3.78 & 0.1 & 46.18 & 24.3 & 9.87 & 4.54 & 1.9 & 40.1 & 21.7 & $L I G$ \\
\hline$a_{24}$ & united-90 & 9.54 & 4.27 & 3.6 & 45.65 & 24.5 & 2.62 & 2.1 & 6.2 & 35.2 & 20.9 & $L I G$ \\
\hline$a_{25}$ & united-91 & 8.57 & 4.08 & 4.6 & 41.2 & 22.5 & 2.61 & 2.01 & 9.8 & 26.6 & 16.2 & $L I G$ \\
\hline$a_{26}$ & united-92 & 6.63 & 3.42 & 5 & 28.59 & 16.6 & 10.09 & 4.1 & 3.3 & 9.9 & 5.8 & $L I G$ \\
\hline$a_{27}$ & usair-88 & 12.33 & 4.76 & 2.7 & 63.93 & 43.6 & 9.22 & 3.48 & 2.7 & 58 & 38.7 & $L I G$ \\
\hline$a_{28}$ & usair-89 & 10.42 & 3.9 & 3.2 & 59.8 & 40.8 & 6.06 & 2.58 & 4.1 & 44.8 & 31.2 & $L I G$ \\
\hline$a_{29}$ & usair-90 & 8.24 & 3.19 & 20 & 52.39 & 35.4 & -1.28 & 0.71 & 20 & 32.3 & 21.8 & $L I G$ \\
\hline$a_{30}$ & alaska-88 & 11.15 & 4 & 3.7 & 59.65 & 34.1 & 10.42 & 5.31 & 2.1 & 63.4 & 42 & $U N I G$ \\
\hline$a_{31}$ & alaska-89 & 10.06 & 3.87 & 4 & 56.68 & 35.2 & 7.24 & 3.86 & 3.5 & 63.7 & 39.1 & $U N I G$ \\
\hline$a_{32}$ & alaska-90 & 10.03 & 4.19 & 3.5 & 55.34 & 35 & 10.42 & 4.73 & 3.1 & 42.4 & 29.1 & $U N I G$ \\
\hline$a_{33}$ & alaska-91 & 8.25 & 3.87 & 4.1 & 54.45 & 34.4 & 6.55 & 2.49 & 7.2 & 34.7 & 23.5 & $U N I G$ \\
\hline$a_{34}$ & alaska-92 & 6.98 & 3.49 & 20 & 45.82 & 30 & 0.29 & 1.07 & 20 & 24.9 & 16.3 & $U N I G$ \\
\hline$a_{35}$ & usair-91 & 6.18 & 2.51 & 20 & 43.86 & 29 & 2.21 & 1.49 & 15.4 & 25.3 & 17.1 & $U N I G$ \\
\hline$a_{36}$ & usair-92 & 5.08 & 2.3 & 20 & 31.27 & 21.2 & 9.21 & 3.23 & 4.2 & -4 & -2.6 & $U N I G$ \\
\hline$a_{37}$ & airwiss-88 & 12.23 & 2.79 & 5.6 & 48.94 & 36.5 & 8.77 & 3.54 & 4.5 & 65.1 & 43.3 & $L N I G$ \\
\hline$a_{38}$ & airwiss-89 & 9.72 & 2.78 & 5.9 & 51.96 & 37.5 & 7.17 & 2.87 & 5.3 & 63.9 & 42.5 & $L N I G$ \\
\hline$a_{39}$ & airwiss-90 & 8.52 & 2.68 & 7.8 & 53.5 & 38.3 & 2.48 & 1.6 & 18.6 & 48.6 & 35.6 & $L N I G$ \\
\hline$a_{40}$ & metro-88 & 9.65 & 5.18 & 3.6 & 46.91 & 32.2 & 4.67 & 2.72 & 6.8 & 28.6 & 17.6 & $L N I G$ \\
\hline$a_{41}$ & panam-88 & 1.64 & 1.42 & 0.5 & 2.46 & 0.4 & 6.49 & 2.88 & 4.3 & -27.2 & -14.2 & $L N I G$ \\
\hline$a_{42}$ & panam-89 & -2.65 & 0.28 & 20 & -10.53 & -6.5 & -16.4 & -0.66 & 20 & -49.7 & -25.8 & $L N I G$ \\
\hline$a_{43}$ & united-88 & 7.76 & 3.79 & 6.5 & 47.41 & 26.3 & 7.95 & 4.33 & 3.6 & 31.1 & 18.3 & $L N I G$ \\
\hline$a_{44}$ & metro-89 & 5.7 & 4.09 & 2.1 & 42.36 & 27.9 & -9.14 & -1.64 & 20 & 24.9 & 13.7 & $S p G$ \\
\hline$a_{45}$ & metro-90 & 2.28 & 2.6 & 0.2 & 30.87 & 20.3 & -3.64 & -0.07 & 20 & 24.9 & 13.7 & $S p G$ \\
\hline$a_{46}$ & panam-90 & -1.38 & 0.58 & 2.4 & -41.67 & -22.52 & 2.37 & 1.57 & 12.4 & -111.3 & -61.6 & $S p G$ \\
\hline$a_{47}$ & ctn-91 & -7.31 & 0.09 & 11.5 & -73.91 & -47.1 & 3.18 & 2.09 & 1.5 & -170.3 & -107.1 & $S p G$ \\
\hline$a_{48}$ & metro-91 & -3.09 & 0.28 & 20 & -18.92 & -4.2 & -17.86 & -5.27 & 0.2 & -189.3 & -82.7 & $S p G$ \\
\hline$a_{49}$ & metro-92 & -4.27 & 0.32 & 0.4 & -56.51 & -23.2 & 4.61 & 0.01 & 7.5 & -138.9 & -60.3 & $S p G$ \\
\hline
\end{tabular}

